\title{
Non-LTE oxygen line formation in 3D hydrodynamic model stellar atmospheres
}

\author{
A. M. Amarsi ${ }^{1 \star}$, M. Asplund ${ }^{1}$, R. Collet $^{1}$, and J. Leenaarts ${ }^{2}$ \\ ${ }^{1}$ Research School of Astronomy and Astrophysics, Australian National University, ACT 2611, Australia \\ 2 Institute for Solar Physics, Stockholm University, SE-106 91 Stockholm, Sweden
}

Accepted 2015 November 03. Received 2015 November 03; in original form 2015 September 07

\begin{abstract}
The OI $777 \mathrm{~nm}$ lines are among the most commonly used diagnostics for the oxygen abundances in the atmospheres of FGK-type stars. However, they form in conditions that are far from local thermodynamic equilibrium (LTE). We explore the departures from LTE of atomic oxygen, and their impact on OI lines, across the STAGGER-grid of three-dimensional hydrodynamic model atmospheres. For the OI $777 \mathrm{~nm}$ triplet we find significant departures from LTE. These departures are larger in stars with larger effective temperatures, smaller surface gravities, and larger oxygen abundances. We present grids of predicted 3D non-LTE based equivalent widths for the OI $616 \mathrm{~nm}$, [OI] $630 \mathrm{~nm},[\mathrm{OI}] 636 \mathrm{~nm}$, and OI $777 \mathrm{~nm}$ lines, as well as abundance corrections to 1D LTE based results.
\end{abstract}

Key words: radiative transfer - line: formation — stars: abundances — stars: atmospheres - methods: numerical

\section{INTRODUCTION}

As the most abundant of the metals, oxygen plays an important role in many areas of astrophysics. It has, for example, a large impact on stellar structure and evolution due to its large opacity contribution and the importance of the CNO nucleosynthesis cycle (e.g. VandenBerg et al. 2012), to the extent that the stipulated errors in the ages of metal poor stars and globular clusters inferred from theoretical evolutionary tracks can be dominated by uncertainties in the oxygen abundances (e.g. Krauss \& Chaboyer 2003; Bond et al. 2013). Oxygen can also have a large impact on the chemistry of exoplanetary atmospheres (Madhusudhan 2012), which is driving interest in determining the carbon-to-oxygen ratio in exoplanet host stars (e.g. Petigura \& Marcy 2011; Nissen et al. 2014). Finally, since oxygen abundances can be measured using different diagnostics in a variety of sites and down to very low metallicities (e.g. Stasińska et al. 2012), they are key tracers of the chemical evolution of our galaxy (e.g. Tinsley 1979; McWilliam 1997).

Abundances of oxygen, and indeed of all chemical species, in the atmospheres of stars cannot be measured directly. They must instead be inferred by matching observations to models. Inadequacies in the models of stellar atmospheres and spectrum formation can cause the extracted abundances to be unrealistic.

In particular, large systematic errors can arise by im-

* E-mail: anish.amarsi@anu.edu.au posing local thermodynamic equilibrium (LTE). It has long been known (Thomas 1957; Pecker 1959; Jefferies 1966; de Jager \& Neven 1967) that the photospheres of late-type stellar atmospheres show significant departures from LTE since the densities of electrons and neutral hydrogen can become so small that energy partitioning according to Boltzmann statistics is no longer maintained (e.g. Mihalas \& Athay 1973). This has direct implications on the strengths of the lines which form there, and thus on the inferred abundances.

Errors can also arise by employing one dimensional (1D) hydrostatic model atmospheres, which neglect atmospheric inhomogeneities, and typically predict, for a given effective temperature and surface gravity, temperature stratifications that are too shallow in the upper atmospheric layers at low $[\mathrm{Fe} / \mathrm{H}]$ (e.g. Asplund et al. 1999) and too steep at solar metallicities (e.g. Pereira et al. 2013) compared to the mean stratification of their 3D counterparts. These effects can couple to the departures from LTE to affect the line formation in complicated ways, and in general do not average out (e.g. Asplund 2005). The inadequacies of $1 \mathrm{D}$ hydrostatic models stem from their intrinsic inability to realistically describe the transport of energy by convection processes and from the lack of self-consistent atmospheric velocity fields (e.g. Collet et al. 2007). In that respect, ab initio 3D hydrodynamic models (e.g. Nordlund et al. 2009; Freytag et al. 2012; Magic et al. 2013a) are far preferable.

In FGK-type stars the OI $777 \mathrm{~nm}$ triplet lines are among the most commonly used oxygen abundance diagnostics, as they are typically strong and free of blends. However, these 
lines form in conditions that depart from LTE. Non-LTE effects were first noted in these lines by Altrock (1968), and later critically studied with multi-level non-LTE radiative transfer techniques by Sedlmayr (1974). For a given abundance the lines were found to be significantly stronger in non-LTE, a conclusion that has been supported by numerous subsequent studies (e.g. Eriksson \& Toft 1979; Kiselman 1993; Gratton et al. 1999; Takeda 2003; Fabbian et al. 2009). Studies of non-LTE effects in three dimensional models have hitherto been restricted to the Sun (Kiselman \& Nordlund 1995; Asplund et al. 2004; Pereira et al. 2009; Prakapavičius et al. 2013; Steffen et al. 2015); such studies were critical for the recent downwards revision of over 0.2 dex of the canonical solar oxygen abundance (cf. Asplund et al. 2004, 2009; Caffau et al. 2008).

Other permitted atomic oxygen lines (in particular the OI $615.8 \mathrm{~nm}$ line) see less use on account of their weakness, the presence of blends and/or the lack of accurate atomic data. They are less susceptible to non-LTE effects than the OI $777 \mathrm{~nm}$ lines (e.g. Asplund et al. 2004; Caffau et al. 2008). The forbidden [OI] $630.0 \mathrm{~nm}$ and [OI] $636.3 \mathrm{~nm}$ lines are a popular alternative (e.g. Lambert 1978) and have the tremendous advantage of forming essentially in LTE (e.g. Asplund et al. 2004). However, they are usually weak, sensitive to the model atmosphere (e.g. Nissen et al. 2002), and severely blended (e.g. Lambert 1978; Allende Prieto et al. 2001).

Molecular oxygen lines, particularly $\mathrm{OH}$ electron excitation lines in the UV, are also popular (e.g. Israelian et al. 1998; Boesgaard et al. 1999). However, to obtain accurate abundances from these lines, they must be modelled in $3 \mathrm{D}$, owing to the steep temperature sensitivity of molecule formation: the abundances inferred from 1D models can be over 0.5 dex too large (Asplund \& García Pérez 2001; Collet et al. 2007). Multi-level non-LTE analyses of OH lines have not yet been attempted, although an exploratory study by Asplund \& García Pérez (2001), who used 1D models and employed the two-level approximation, found that the nonLTE abundances were lowered by as much as $0.25 \mathrm{dex}$. NonLTE analyses of these lines are limited by the lack of accurate collisional rate coefficients and by the computational resources that they demand; thus, full 3D non-LTE analyses of molecular oxygen lines still await to be tackled.

In this paper we detail recent $3 \mathrm{D}$ multi-level non-LTE atomic oxygen line formation calculations across a grid of 3D hydrodynamic STAGger stellar model atmospheres. After describing the simulation setup in Sect. 2, we present the results, including a description of the non-LTE mechanism, the 3D effects, and abundance errors, in Sect. 3. We focus our discussion on the OI $777 \mathrm{~nm}$ lines, although we discuss abundance errors for the OI $615.8 \mathrm{~nm}$, [OI] $630.0 \mathrm{~nm}$ and [OI] $636.4 \mathrm{~nm}$ lines as well. We compare our results with those from related studies in Sect. 4 before summarising in Sect. 5 .

\section{METHOD}

\subsection{Overview}

We briefly review the governing equations of non-LTE radiative transfer (e.g. Rutten 2003; Hubeny \& Mihalas 2014).
Non-LTE analyses typically assume statistical equilibrium,

$$
\sum_{j \neq i}\left(n_{j} P_{j i}-n_{i} P_{i j}\right)=0
$$

where the transition rate from energy level $i$ to energy level $j$ (which have corresponding populations $n_{i}$ and $n_{j}$ ) can be split into a radiative component and a collisional component: $P_{i j}=R_{i j}+C_{i j}$. This equation states that in equilibrium, the flow into an arbitrary energy level is balanced by the flow out of it: $\frac{\mathrm{d} n_{i}}{\mathrm{~d} t}=0$. The system of equations is closed by conserving the total number density: $\sum_{i} n_{i}=N$. In stellar photospheres, particle velocities have to a good approximation Maxwell-Boltzmann distributions (due to the efficiency of electron collisions in thermalising the medium; see Hubeny \& Mihalas 2014, and references therein). Thus, collisions tend to drive levels to be in relative LTE. In the special case where collisional rates dominate over all of the radiative rates, LTE is reproduced.

To obtain the radiative rates, the radiation field must first be determined by solving the radiative transport equation for the specific intensity $I_{\nu}$ along a ray,

$$
\frac{\mathrm{d} I_{\nu}}{\mathrm{d} s}=\alpha_{\nu}\left(S_{\nu}-I_{\nu}\right)
$$

where $s$ is the path length (e.g. Rutten 2003). The linear extinction coefficient $\alpha_{\nu}$ and source function $S_{\nu}$ are functions of the populations, making the problem strongly non-linear. Radiative transfer couples different parts of the atmosphere together, making the problem a non-local one. Through the radiation field, all levels are in principle coupled to each other, everywhere in the atmosphere.

\subsection{Code description}

The system of equations discussed in Sect. 2.1 was solved iteratively by the MPI-parallelised domain-decomposed 3D non-LTE radiative transfer code Multi3D (Leenaarts \& Carlsson 2009). Details of the code can be found in that paper and references therein; we briefly discuss some of its important aspects, as well as recent changes made to the code.

Multi3D accepts 3D model atmospheres defined on a Cartesian grid that is periodic and has equidistant spacing in the two horizontal dimensions. In the vertical direction the grid can be non-equidistant. The model atmospheres specify the gas temperature $T$, gas density $\rho$ and electron number density $n_{\mathrm{e}}$. The populations of all species not treated in nonLTE are computed from these three thermodynamic variables assuming LTE. Importantly, the hydrodynamic models predict the velocity field $\boldsymbol{v}$, which is used by MuLti3D to find the Doppler shifts introduced by macroscopic gas flows at every gridpoint. The species being modelled is assumed to be a trace element having no feedback on the background model atmosphere. This is a good approximation for oxygen which does not contribute significantly to the continuous opacity, nor to the number of free electrons.

The system of equations is solved using the Multilevel Approximate Lambda Iteration (MALI) preconditioning method of Rybicki \& Hummer (1991, 1992) (see also Uitenbroek 2001), in which, expressing the solution to Eq. 2 as $I_{\nu}=\Psi_{\nu}\left[\eta_{\nu}\right]$, the $\Psi_{\nu}$ operator is preconditioned using an 
approximate operator $\Psi_{\nu}^{*}$

$$
I_{\nu}=\Psi_{\nu}^{*}\left[\eta_{\nu}\right]+\left(\Psi_{\nu}-\Psi_{\nu}^{*}\right)\left[\eta_{\nu}^{\dagger}\right] .
$$

Here $\eta_{\nu}$ is the emissivity and $\eta_{\nu}^{\dagger}$ is the emissivity determined using the populations of the previous iteration. Multi3D uses the diagonal of the $\Psi_{\nu}$ operator as the approximate operator.

The formal solution (the evaluation of Eq. 3) is performed on short characteristics (the integral form of the radiative transfer equation is solved analytically piecewise along the ray; Olson \& Kunasz 1987) using cubicconvolutuion interpolation of the upwind and downwind quantities, and cubic Hermite spline interpolation for the source function (Auer 2003; Fabiani Bendicho 2003; Ibgui et al. 2013). At the lower boundary, the upwards intensity field is taken to be that of a blackbody at the local material temperature, while at the upper boundary it is assumed that there is no incident radiation. Complete redistribution is assumed in the lines and continua of the non-LTE atom, and isotropic coherent scattering in all background processes. The angle-averaged radiation field is calculated for 24 different directions using Carlson's quadrature set A4 (Carlson 1963; Bruls et al. 1999).

Acceleration of convergence techniques are important both for achieving acceptable convergence rates and for avoiding stabilisation of the solution (e.g. Auer 1991, compare Fig. 3 and Fig. 4.) The algorithm of Kao \& Auer (1990, Appendix) was adopted, which minimizes the global residual in the populations with respect to a set of conjugate vectors between iterations. We note briefly that their method is analogous to the generalized conjugate residual algorithm $\operatorname{gcr}(m)$, with restart parameter $m=2$, that is presented in Saad (2003) in the context of solving linear systems of equations.

Once the populations have converged, a final formal solution is performed to find the emergent specific intensities. For this final formal solution, interpolation of intensities is avoided in order to reduce the numerical diffusion in the solution (long-characteristics; e.g. Auer 2003). This is achieved through horizontal interpolation of the extinction coefficients and source functions onto the rays, for every layer of the model atmosphere and every oblique ray. The emergent intensity can then be found immediately by direct integration. The final emergent intensities $\bar{I}_{\nu}$ are computed by averaging $I_{\nu}$ over the horizontal and temporal dimensions using equidistant trapezoidal integration. The emergent specific continuum intensity $\bar{I}_{\nu}^{\mathrm{c}}$ are obtained in a similar fashion, at each frequency point, but with zero contribution to the extinction and source function from lines.

The emergent flux (i.e. the disk-integrated intensity)

$$
\bar{F}_{\nu}=\int_{0}^{2 \pi} \int_{0}^{1} \bar{I}_{\nu} \mu \mathrm{d} \mu \mathrm{d} \phi,
$$

and the emergent continuum flux,

$$
\bar{F}_{\nu}^{\mathrm{c}}=\int_{0}^{2 \pi} \int_{0}^{1} \bar{I}_{\nu}^{\mathrm{c}} \mu \mathrm{d} \mu \mathrm{d} \phi,
$$

are calculated using $n_{\mu}$-point Lobatto quadrature for the integral over $\mu$ on the interval [0,1] (e.g. Hildebrand 1956), and equidistant $n_{\phi}$-point trapezoidal integration for the integral over $\phi$. We used $n_{\mu}=5$ and, for $\mu \neq 0$ and $\mu \neq 1$, we used $n_{\phi}=4$. When $\mu=0$, the integrand is identically nought and

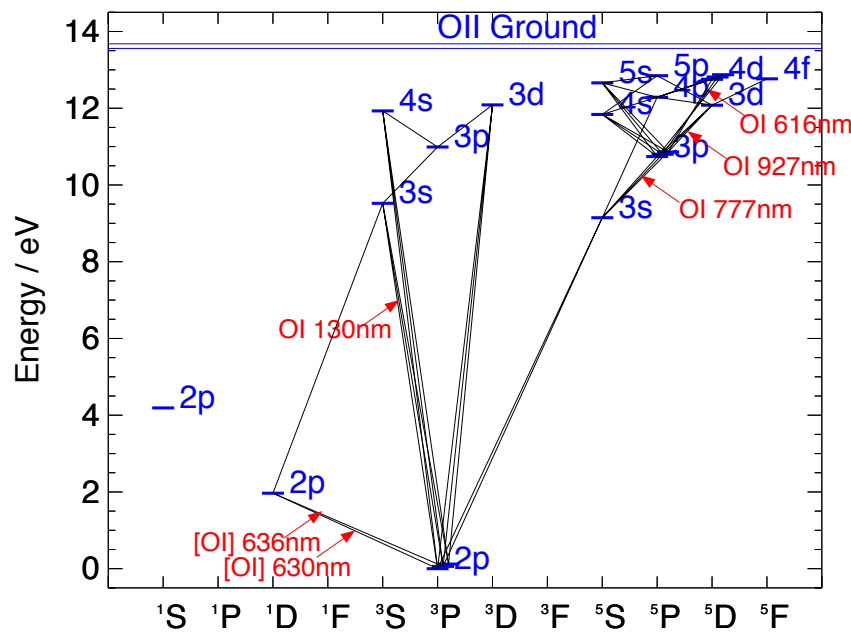

Figure 1. Grotrian diagram of the 22 levels of oxygen and the 43 radiative transitions included in the model atom. Fine structure has been magnified. The ground state of OII is also included in the model atom, along with 22 bound-free radiative transitions (not depicted).

the formal solution is not performed, whilst when $\mu=1$ the ray is vertical and only a single formal solution is required. We note briefly that, because of these properties of the integrand, for a given number of formal solutions Lobatto quadrature is of higher nominal accuracy than the closely related Gaussian quadratures and Radau quadratures (e.g. Hildebrand 1956).

\subsection{Background opacities}

Continuous background opacities were calculated at runtime everywhere in the 3D stellar atmosphere using the Uppsala opacity package (Gustafsson et al. 1975, and subsequent updates). These opacities were calculated under the assumption of LTE. Oxygen continua that were also present in the model atom (discussed below) were not included at this point.

Background line opacities in the UV were precomputed, in pure absorption and assuming LTE, on a grid of gas temperatures and pressures and with an assumed equation of state for the appropriate chemical compositions of the models (Collet et al. 2005; Bergemann et al. 2012). The line opacities were interpolated onto the model atmosphere by MuLTi3D. In particular, the $121.5 \mathrm{~nm}$ Lyman- $\alpha$ transition was included as a background opacity in this way; we found this line to have a dramatic effect on the statistical equilibrium at low metallicities (see Sect. 3.4).

\subsection{Model atom}

The model oxygen atom that was used in this work is based on those used by Carlsson \& Judge (1993), Kiselman (1993), and Fabbian et al. (2009). We show in Fig. 1 the radiative transitions connecting the 23 levels (22 excited levels plus 1 ionised level) included in the model. The model was updated to include energies and Einstein coefficients 
Table 1. Collisional transitions included in the model atom. Charge transfer was only included for the oxygen ground state. Collisional rate coefficients $q$ are obtained from collisional cross-sections $\sigma$ via the relation $q=<\sigma v>$; here the brackets indicate averaging over the Maxwell-Boltzmann distribution for speeds $v$. Assuming that the particle speeds have Maxwell-Boltzmann distributions, the forward and reverse rate coefficients are related by the Boltzmann factor (e.g. Rutten 2003).

\begin{tabular}{cccccc}
\hline Transition & & Equation & Reference & Comment \\
\hline \hline Electron excitation & $\mathrm{O}+\mathrm{e}^{-}$ & $\longleftrightarrow$ & $\mathrm{O}^{*}+\mathrm{e}^{-}$ & Barklem (2007) & Ab initio; quantum mechanical \\
\hline Electron ionisation & $\mathrm{O}+\mathrm{e}^{-}$ & $\longleftrightarrow$ & $\mathrm{O}^{+}+2 \mathrm{e}^{-}$ & Allen (1973) & Empirical \\
\hline Neutral hydrogen excitation & $\mathrm{O}+\mathrm{H}$ & $\longleftrightarrow$ & $\mathrm{O}^{*}+\mathrm{H}$ & Lambert (1993) & Semi-empirical; semi-classical \\
\hline Neutral hydrogen ionisation & $\mathrm{O}+\mathrm{H}$ & $\longleftrightarrow$ & $\mathrm{O}^{+}+\mathrm{H}+\mathrm{e}^{-}$ & Lambert (1993) & Semi-empirical; semi-classical \\
\hline Proton charge transfer & $\mathrm{O}+\mathrm{H}^{+}$ & $\longleftrightarrow$ & $\mathrm{O}^{+}+\mathrm{H}$ & Stancil et al. (1999) & Mixed \\
\hline
\end{tabular}

from NIST $^{1}$ (Kramida et al. 2015), natural line broadening coefficients from VALD3 ${ }^{2}$ (Piskunov et al. 1995; Heiter et al. 2008), and collisional line broadening coefficients from Barklem et al. (1998). Photoionisation cross-sections were the same as those used in the papers cited above; i.e. taken from the Opacity Project (Cunto et al. 1993), or calculated semi-empirically (Peach 1971). Oscillator strengths for the [OI] $630 \mathrm{~nm}$ and [OI] $636 \mathrm{~nm}$ lines were taken from (Storey \& Zeippen 2000), and atomic data for the Ni blend in the former line were taken from Johansson et al. (2003).

We show in Table 1 the collisional transitions included in the model and their sources. The collisional rates between fine structure levels were set to very large values, which ensured that the populations within the fine structure were distributed according to their statistical weights (e.g. Kiselman 1993). Following Lambert (1993), a correction factor $S_{\mathrm{H}}$ to the original formula of Drawin $(1968,1969)$ was adopted to describe neutral hydrogen collisions. Levels with radiatively weak transitions were coupled using an effective oscillator strength $f_{\min }=10^{-3}$. Following Pereira et al. (2009), and using their observational data from the Swedish 1-m Solar Telescope (Scharmer et al. 2003), the centre-to-limb variation in the Sun of the OI $777 \mathrm{~nm}$ lines were used to calibrate $S_{\mathrm{H}}$. We obtained $S_{\mathrm{H}} \approx 1$ and inferred a solar oxygen abundance $\log \epsilon_{\mathrm{O}}^{\ominus} \approx 8.7$. A detailed description of the fitting procedure will be presented in a forthcoming paper.

\subsection{Model atmospheres}

Line formation calculations were performed across a grid of 3D hydrodynamic STAGger model atmospheres (Collet et al. 2011; Magic et al. 2013a). Each model in the grid is specified by a nominal effective temperature $T_{\text {eff }}$, surface gravity $\log g^{3}$, and $[\mathrm{Fe} / \mathrm{H}]^{4}$. Solar scaled abundances (Asplund et al. 2009) were adopted, with $\alpha$-enhancement $[\alpha / \mathrm{Fe}]=0.4$ for models with $[\mathrm{Fe} / \mathrm{H}] \leq-1.0$. Since oxygen was treated as a trace element, the oxygen abundance was

1 http://www.nist.gov/pml/data/asd.cfm

2 http://vald.astro.uu.se/ vald/php/vald.php

3 Here and henceforth, $\log (\ldots) \equiv \log _{10}(\ldots)$.

4 The logarithmic abundance of an arbitrary element $\mathrm{A}$ is defined with respect to hydrogen: $\log \epsilon_{\mathrm{A}}=\log \frac{N_{\mathrm{A}}}{N_{\mathrm{H}}}+12$. The abundance ratio of elements $\mathrm{A}$ and $\mathrm{B}$ is given by: $[\mathrm{A} / \mathrm{B}]=\left(\log \epsilon_{\mathrm{A}}-\log \epsilon_{\mathrm{A}}^{\odot}\right)-$ $\left(\log \epsilon_{\mathrm{B}}-\log \epsilon_{\mathrm{B}}^{\odot}\right)$, where $\odot$ denotes the solar value. varied independently. Calculations were performed on four snapshots for each 3D model, equally spaced across a time sequence spanning approximately two convective turnover times (Magic et al. 2013a).

The original models have Cartesian geometry with $x y z-$ grid size $240 \times 240 \times 240$. These were modified in several steps before performing the detailed line formation calculations. First, every other gridpoint in the horizontal dimensions was dropped, reducing the number of gridpoints by a factor of four. Second, unphysical ghost layers (5 layers on both the upper and the lower boundaries) were removed as well as additional layers such that the top-most layer satisfied $\operatorname{Max}\left(\log \tau_{500}\right) \lesssim-5$ and that the bottom-most layer satisfied $\operatorname{Min}\left(\log \tau_{500}\right) \gtrsim 3$, where $\log \tau_{500}$ is the vertical optical depth at wavelength $\lambda=500 \mathrm{~nm}$. Finally, $T, \log \rho$, $\log n_{\mathrm{e}}$ and $\boldsymbol{v}$ were interpolated using cubic splines onto a new vertical grid. This new vertical grid was constructed by demanding that the horizontal-mean step in $\log \tau_{500}$ was roughly constant between layers. The final grid size was fixed at $120 \times 120 \times 110$ (where the last dimension represents the vertical).

Line formation calculations were also performed on $1 \mathrm{D}$ plane-parallel hydrostatic Aтмо (Magic et al. 2013a, Appendix A) model atmospheres. These models were computed using an equation of state and a treatment of opacities and radiative transfer fully consistent with the STAGGER models. They have 110 depth points and span $-5 \leq \log \tau_{\mathrm{R}} \leq 3$, where $\log \tau_{\mathrm{R}}$ is the logarithmic vertical Rosseland mean optical depth. The models use mixing length theory (BöhmVitense 1958) to account for convective energy transport in an approximate way. The adopted mixing length parameter was $\alpha_{\mathrm{MLT}}=1.5$ (in units of pressure scale heights). This parameter affects the atmospheric structure in the deepest layers, below where the relevant oxygen lines form; hence, the results are insensitive to the precise value of this parameter. The original opacity sampling data were computed assuming a microturbulence parameter of $1.0 \mathrm{~km} \mathrm{~s}^{-1}$; these opacities are sorted into 12 bins in exactly the same way as for the corresponding 3D STAGGER simulations. Apart from a different choice of angle quadrature for the mean intensity (10 directions, based on 5-point Gaussian quadrature on the interval $\mu \in[0,1])$ and the use of another microturbulence parameter $\xi$ (discussed in Sect. 2.6), the 1D line formation calculations proceeded in the same way as the $3 \mathrm{D}$ calculations using Multi3D. 
Table 2. Nominal effective temperatures, and corresponding surface gravities, on the nodes on the grid.

\begin{tabular}{cccccc}
\hline Nominal $T_{\text {eff }} / \mathrm{K}$ & \multicolumn{5}{c}{$\log \left(\mathrm{g} / \mathrm{cm} \mathrm{s}^{-2}\right)$} \\
\hline \hline 5000 & 3.0 & 3.5 & 4.0 & 4.5 & 5.0 \\
\hline 5500 & 3.0 & 3.5 & 4.0 & 4.5 & 5.0 \\
\hline 6000 & & 3.5 & 4.0 & 4.5 & \\
\hline 6500 & & & 4.0 & 4.5 & \\
\hline
\end{tabular}

Table 3. Background chemical compositions, and corresponding non-LTE oxygen abundances, on the nodes of the grid. LTE runs were performed for all oxygen abundances $5.7 \leq \log \epsilon_{\mathrm{O}} \leq 10.7$ in regular intervals of 0.5 dex.

\begin{tabular}{cccccccccc}
\hline$[\mathrm{Fe} / \mathrm{H}]$ & \multicolumn{10}{c}{$\log \epsilon_{\mathrm{O}}$} \\
\hline \hline-3.0 & 5.7 & 6.2 & 6.7 & 7.2 & 7.7 & & & & \\
\hline-2.0 & 5.7 & 6.2 & 6.7 & 7.2 & 7.7 & 8.2 & 8.7 & & \\
\hline-1.0 & & & 6.7 & 7.2 & 7.7 & 8.2 & 8.7 & 9.2 & \\
\hline 0.0 & & & & & 7.7 & 8.2 & 8.7 & 9.2 & 9.7 \\
\hline
\end{tabular}

2.6 Equivalent widths, abundance corrections and abundance errors

To obtain equivalent widths, the non-LTE/LTE ratios of the averaged emergent intensities from MuLTI3D were found and applied to the 3D LTE averaged emergent intensities calculated using SCATE (Hayek et al. 2010) over at least 50 temporal snapshots across the entire time sequence. The intensities were averaged not just over the spatial and temporal dimensions, but also over the the azimuthal angle, using equidistant trapezoidal integration. The flux was then computed using Eq. 4. This procedure was repeated using the continuum intensity (Eq. 5); thereby the normalized flux could be obtained. It is better to use intensity ratios at this stage instead of flux ratios; if the systematic errors associated with using just four snapshots have any $\mu$ dependence, the error in the flux ratio will be larger than the error in the intensity ratios. The equivalent widths were found by direct integration.

We define abundance corrections as the abundance differences compared to 1D LTE. For example, the 3D non-LTE abundance correction,

$$
\Delta_{1 \mathrm{~L}}^{3 \mathrm{~N}}\left(\log \epsilon_{\mathrm{O}}^{1 \mathrm{D}, \mathrm{LTE}}\right)=\log \epsilon_{\mathrm{O}}^{3 \mathrm{D}, \mathrm{NLTE}}-\log \epsilon_{\mathrm{O}}^{1 \mathrm{D}, \mathrm{LTE}},
$$

is determined by demanding that the equivalent width of the line flux obtained in LTE from the 1D model with a given oxygen abundance $\left(\log \epsilon_{\mathrm{O}}^{1 \mathrm{D}, \mathrm{LTE}}\right)$ matches that obtained from the $3 \mathrm{D}$ non-LTE grid with some oxygen abundance $\left(\log \epsilon_{\mathrm{O}}^{3 \mathrm{D}, \mathrm{NLTE}}\right)$. It is a function of the $1 \mathrm{D}$ LTE abundance and the stellar parameters. In contrast, we define abundance errors as the abundance differences compared to 3D nonLTE. For example, the 1D LTE abundance error,

$$
\Delta_{3 \mathrm{~N}}^{1 \mathrm{~L}}\left(\log \epsilon_{\mathrm{O}}^{3 \mathrm{D}, \mathrm{NLTE}}\right)=\log \epsilon_{\mathrm{O}}^{1 \mathrm{D}, \mathrm{LTE}}-\log \epsilon_{\mathrm{O}}^{3 \mathrm{D}, \mathrm{NLTE}},
$$

is a function of the 3D non-LTE abundance and the stellar parameters. Abundance errors are more illuminating in the context of this work and are discussed in Sect. 3.6. However, abundance corrections to 1D LTE based results are more immediately applicable to surveys of FGK-type stars; such corrections have already been illustrated in a previous publication (Amarsi et al. 2015), and are tabulated in this work in Sect. 3.7.

The extent of the grids is summarised in Table 2 and Table 3. The actual (time-averaged) effective temperatures of the 3D hydrodynamic simulations are not input parameters, and for a given simulation is slightly different to its nominal effective temperature (Magic et al. 2013a). The effective temperatures of the $1 \mathrm{D}$ models were set to match those of their 3D counterparts. With the 1D models, line formation calculations were performed using microturbulence parameters $\xi=0.5,1.0,1.5$ and $2.0 \mathrm{~km} \mathrm{~s}^{-1}$. This parameter is necessary to account for the line broadening by convective velocity fields, which are not predicted in the $1 \mathrm{D}$ models.

\section{RESULTS}

\subsection{Non-LTE effects}

The departure coefficients i.e. the ratios of the non-LTE statistical equilibrium populations to the LTE populations,

$$
b_{i}=\frac{n_{i}}{n_{i}^{*}},
$$

are quantitative measures of the departures from LTE. In the Wien regime, the line source function approximately scales with $\frac{b_{u}}{b_{l}}$ and the line extinction approximately scales with $b_{l}$ (e.g. Rutten 2003). Used together with line contribution functions (e.g. Magain 1986; Albrow \& Cottrell 1996; Amarsi 2015), which can be used to identify the line forming regions, the departure coefficients are illuminating and intuitive diagnostics of non-LTE line formation.

We plot in Fig. 2 the probability distributions of the gas temperature, the non-LTE frequency-integrated contribution function for the absolute line flux depression (Amarsi 2015), and the departure coefficients of the $3 \mathrm{p}{ }^{5} \mathrm{P}$ level and the $3 \mathrm{~s}{ }^{5} \mathrm{~S}$ level (i.e. the upper and lower levels of the OI $777 \mathrm{~nm}$ lines) across the atmosphere. We show this for four representative theoretical stars: a turn-off star and a dwarf, each with $[\mathrm{Fe} / \mathrm{H}]=0.0$ and $[\mathrm{Fe} / \mathrm{H}]=-3.0$.

At large depths, densities are large enough to guarantee that collisional rates dominate over all radiative rates, such that the populations of all levels do not depart significantly from their LTE values. Higher up in the atmosphere, the OI $777 \mathrm{~nm}$ lines form (second row of Fig. 2), and the 3p ${ }^{5} \mathrm{P}$ level and the $3 \mathrm{~s}{ }^{5} \mathrm{~S}$ level populations show departures from their LTE values. This reflects the fact that photon losses in the lines themselves drive the non-LTE effects in these levels (e.g. Asplund 2005), as discussed below.

For the stars shown, as well as more generally across this range of stellar parameters, the $3 \mathrm{~s}{ }^{5} \mathrm{~S}$ level develops a significant overpopulation with respect to LTE in the line-forming regions. As the atmosphere becomes less homogeneous, the spread of departure coefficients on surfaces of equal optical depth becomes greater with height. The behaviour of the $3 \mathrm{p}{ }^{5} \mathrm{P}$ level is more complicated. Generally, in the line 
Solar metallicity turn-off Metal poor turn-off Solar metallicity dwarf $\quad$ Metal poor dwarf

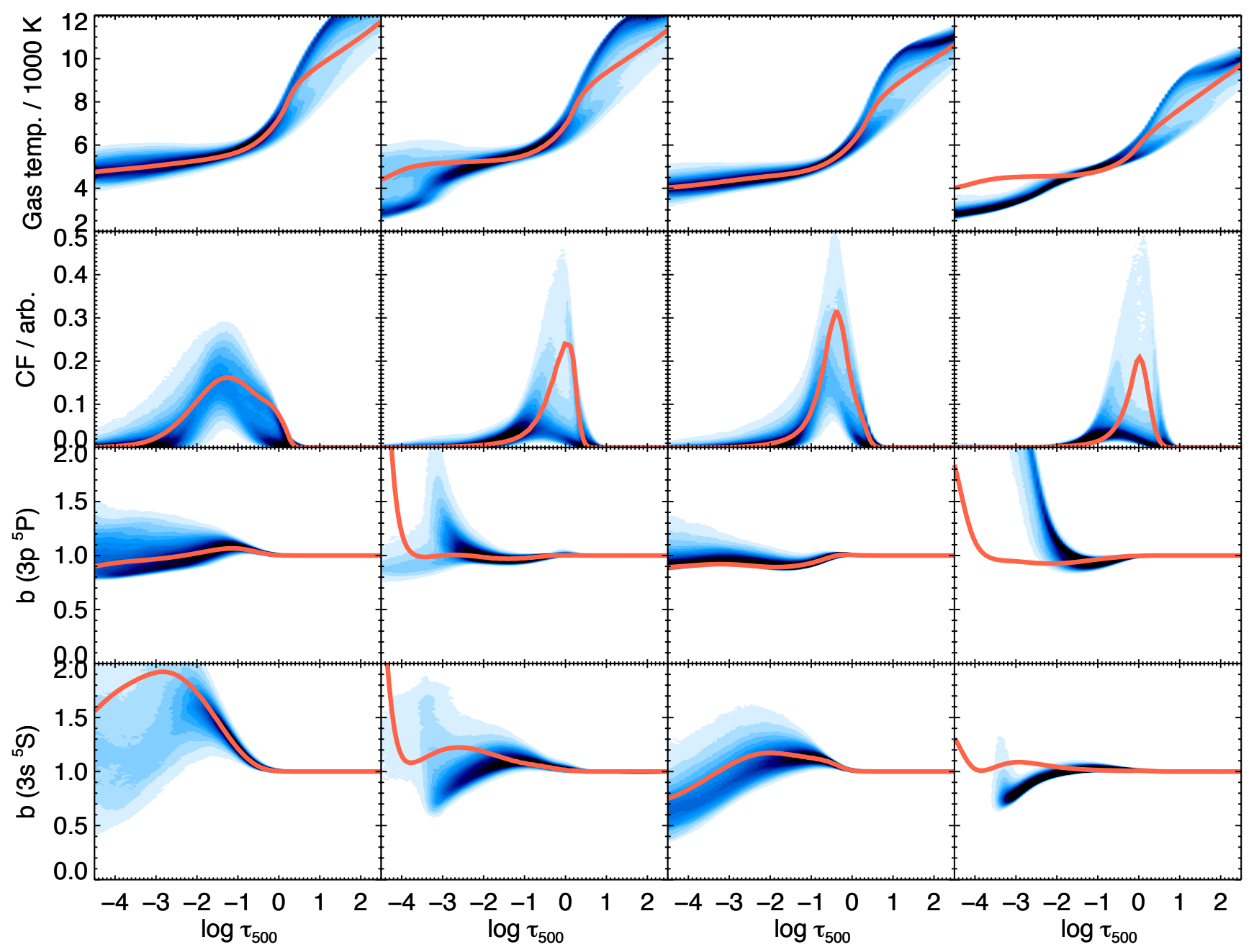

Figure 2. The departures from LTE of the OI $777 \mathrm{~nm}$ lines at different depths in a turn-off $\operatorname{star}\left(T_{\text {eff }} \approx 6500 \mathrm{~K}, \log \left(\mathrm{g} / \mathrm{cm} \mathrm{s}^{-2}\right)=4.0\right)$ and in a dwarf $\left(T_{\text {eff }} \approx 5500 \mathrm{~K}, \log \left(\mathrm{g} / \mathrm{cm} \mathrm{s}^{-2}\right)=4.0\right)$ with $[\mathrm{Fe} / \mathrm{H}]=0.0, \log \epsilon_{\mathrm{O}}=8.7$ (solar metallicity) and $[\mathrm{Fe} / \mathrm{H}]=-3.0, \log \epsilon_{\mathrm{O}}=6.2$ (metal poor). The quantities from top to bottom are gas temperature, non-LTE frequency-integrated contribution function for the absolute line flux depression (in arbitrary units), departure coefficient of the $3 \mathrm{p}^{5} \mathrm{P}$ level (the upper level of the OI $777 \mathrm{~nm}$ lines), and departure coefficient of the $3 \mathrm{~s}{ }^{5} \mathrm{~S}$ level (the lower level of the OI $777 \mathrm{~nm}$ lines). These quantities were evaluated on surfaces of constant $\log \tau_{500}$. Corresponding quantities from the $1 \mathrm{D}$ models are overplotted (red solid lines).

forming regions, the $3 \mathrm{p}{ }^{5} \mathrm{P}$ level stays close to unity or develops a slight underpopulation with respect to LTE. The exception is in stars with high effective temperatures, low surface gravities and higher oxygen abundances (e.g. the solar metallicity turn-off star in the first column of Fig. 2), where the line forming regions are quite extended. In such stars, around the continuum forming layers the $3 \mathrm{p}{ }^{5} \mathrm{P}$ level develops a slight overpopulation with respect to LTE, while higher up, it typically develops an underpopulation with respect to LTE.

Across the 3D STAGGER grid, the overpopulations of the $3 \mathrm{~s}{ }^{5} \mathrm{~S}$ level tend to be larger than the departures from LTE in the $3 p{ }^{5} \mathrm{P}$ level. Therefore in general, the line opacity is increased, while the line source function is decreased, compared to the LTE case. Both of these effects increase the line contribution function and hence the strength of the OI $777 \mathrm{~nm}$ lines with respect to LTE.

To understand the origin of these various effects, the behaviours of all of the energy levels need to be considered. We show on the first row of Fig. 3 the median departure coefficients for all levels across the same set of stars. The OI ground level $\left(2 \mathrm{p}{ }^{3} \mathrm{~S}\right)$ does not deviate significantly from its LTE population throughout the atmosphere, as expected given that oxygen remains overwhelmingly neutral. The efficient charge transfer reaction ensures that the OII ground level also maintains its LTE population. Similarly, the first two excited levels $2 \mathrm{p}{ }^{1} \mathrm{~S}$ and $2 \mathrm{p}{ }^{1} \mathrm{D}$ do not deviate significantly from their LTE populations, due to efficient collisional coupling with the ground level and the lack of any strong radiative couplings. The $3 \mathrm{p}{ }^{5} \mathrm{P}$ level (the upper level 


\section{Solar metallicity turn-off Metal poor turn-off Solar metallicity dwarf Metal poor dwarf}

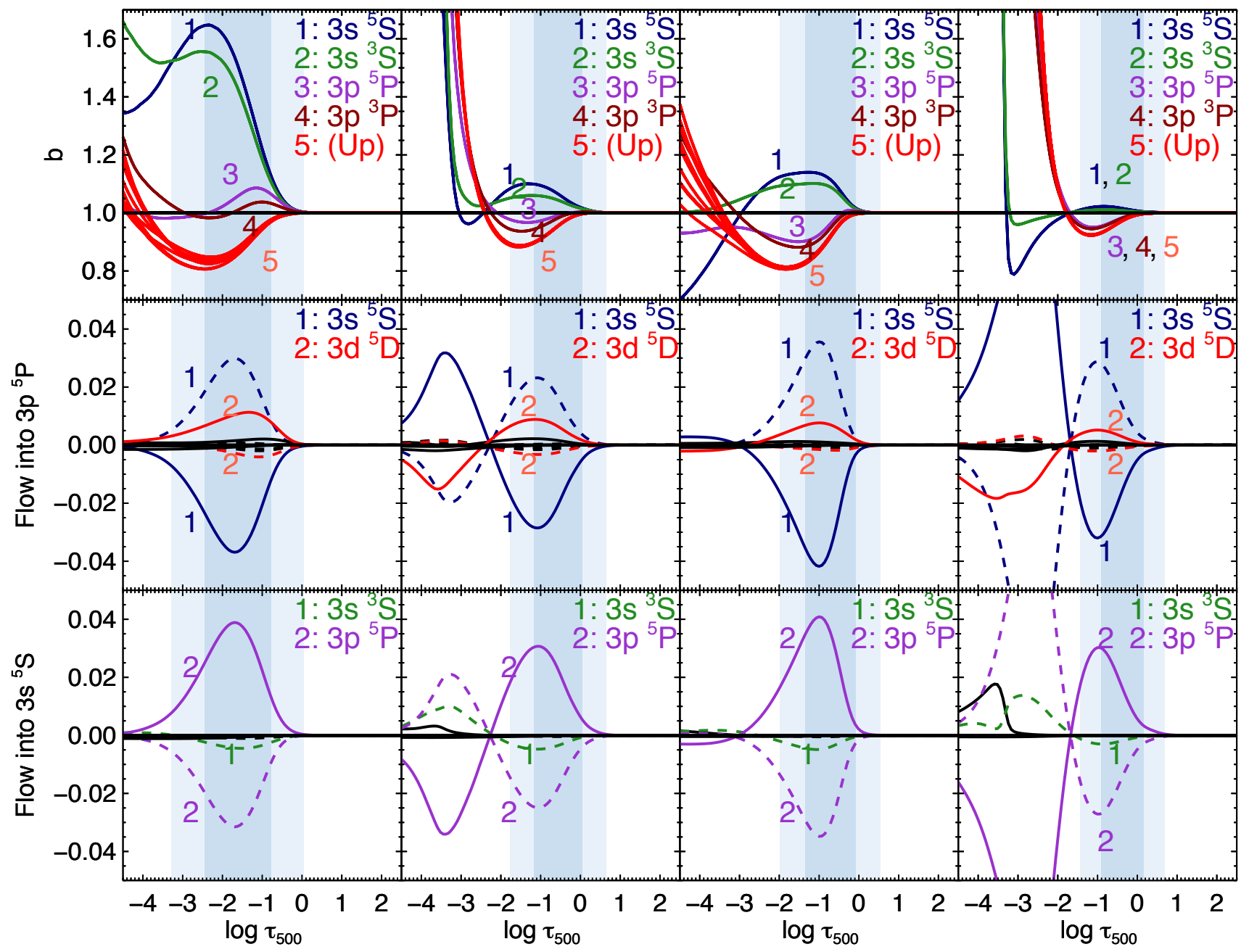

Figure 3. The statistical equilibrium state at different depths in a turn-off star $\left(T_{\text {eff }} \approx 6500 \mathrm{~K}, \log \left(\mathrm{g} / \mathrm{cm} \mathrm{s}^{-2}\right)=4.0\right)$ and in a dwarf $\operatorname{star}\left(T_{\text {eff }} \approx 5500 \mathrm{~K}, \log \left(g / \mathrm{cm} \mathrm{s}^{-2}\right)=4.0\right)$ with $[\mathrm{Fe} / \mathrm{H}]=0.0, \log \epsilon_{\mathrm{O}}=8.7$ (solar metallicity) and $[\mathrm{Fe} / \mathrm{H}]=-3.0, \log \epsilon_{\mathrm{O}}=6.2(\mathrm{metal}$ poor). The top row shows the departure coefficients of each level; the $2 p$ levels in the spin- 0 and spin- 1 systems as well as the OII ground level do not experience departures from their LTE populations and are not shown here. 'Up' refers to the $4 \mathrm{~s}{ }^{5} \mathrm{~S}$ level, and higher energy levels. The second and third rows show the normalized net flow into the $3 \mathrm{p}{ }^{5} \mathrm{P}$ level (the upper level of the OI $777 \mathrm{~nm}$ lines) and the normalized net flow into into the $3 \mathrm{~s}^{5} \mathrm{~S}$ level (the lower level of the OI $777 \mathrm{~nm}$ lines). Collisional transitions are indicated as dashed lines while radiative transitions are indicated as solid lines. Plotted here are median values on surfaces of equal $\log \tau_{500}$. The shaded areas correspond to the mean formation depth \pm one standard deviation, and \pm two standard deviations. The mean formation depth is defined here as the expectation of $\log \tau_{500}$ with respect to the contribution function $C$ in the 3D volume $V: \mathbf{E}\left[\log \tau_{500}\right]=\frac{\int \log \tau_{500} C \mathrm{~d} V}{\int C \mathrm{~d} V}$. The standard deviation is given by $\sqrt{\mathbf{E}\left[\log \tau_{500}^{2}\right]-\mathbf{E}\left[\log \tau_{500}\right]^{2}}$.

of the OI $777 \mathrm{~nm}$ lines) and the $3 \mathrm{p}^{3} \mathrm{P}$ level (its spin-one analogue) behave similarly to each other, as do the $3 \mathrm{~s}{ }^{5} \mathrm{~S}$ level (the lower level of the OI $777 \mathrm{~nm}$ lines) and the $3 \mathrm{~s}{ }^{3} \mathrm{~S}$ level (its spin-one analogue), as a result of efficient intersystem coupling by electron collisions (Barklem 2007). The highly excited upper levels ( $4 \mathrm{~s}{ }^{5} \mathrm{~S}$ and above) are efficiently coupled by neutral hydrogen collisions, thus their departure coefficients behave similarly to each other; they tend to be underpopulated in the line forming regions, for reasons discussed below.

The departure coefficients can in turn be understood by considering the net flow into level $j$ from level $i$ :

$$
\rho_{i j}^{T}=n_{i} T_{i j}-n_{j} T_{j i},
$$

with $T_{i j}=R_{i j}$ or $T_{i j}=C_{i j}$ for radiative and collisional transitions, respectively. In detailed balance, $\rho_{i j}^{T}$ is zero for all values of $i$ and $j$.

We show on the second and third rows of Fig. 3 the median net flow into the $3 \mathrm{p}{ }^{5} \mathrm{P}$ level and into the $3 \mathrm{~s}{ }^{5} \mathrm{~S}$ level for radiative and collisional transitions, normalized using the total flow into those respective levels. In the line forming regions, the net flow is largely dictated by the radiative cou- 
pling between these levels (i.e. the OI $777 \mathrm{~nm}$ lines), balanced by the collisional coupling between these levels. The OI $777 \mathrm{~nm}$ lines act to shift the equilibrium away from LTE to one where the $3 \mathrm{p}{ }^{5} \mathrm{P}$ level is underpopulated and the $3 \mathrm{~s}$ ${ }^{5} \mathrm{~S}$ level is overpopulated.

The mechanism for this is photon losses. Photons that are absorbed by the lines are either destroyed via collisional de-excitation (true absorption), or are scattered, via spontaneous or by stimulated photoemission (e.g. Rutten 2003). Scattered photons can propagate large distances through the atmosphere before being absorbed again (and possibly scattered again), or can escape from the atmosphere altogether. Consequently, there are fewer photoexcitations than there would be given a thermal radiation field, so that the upper level becomes underpopulated and the lower level becomes overpopulated, relative to LTE. This mechanism is particularly effective for the OI $777 \mathrm{~nm}$ lines, which have extended Lorentzian wings. Photons absorbed at the linecentre, where the extinction probability is large, can be scattered into the wings (since complete redistribution is assumed); the extinction probability in the wings is small, so such photons are very likely to propagate large distances and escape. For more details on this mechanism see e.g. chapter 14 of Hubeny \& Mihalas (2014).

Other radiative transitions are also important to the behaviours of the $3 \mathrm{~s}{ }^{5} \mathrm{~S}$ and $3 \mathrm{p}{ }^{5}$ Plevels. In particular, there is a cascade from the collisionally-coupled highly excited upper levels down to the $3 \mathrm{p}{ }^{5} \mathrm{P}$ level (and hence to the $3 \mathrm{~s}{ }^{5} \mathrm{~S}$ level, via the OI $777 \mathrm{~nm}$ lines) via mainly the $3 \mathrm{~d}{ }^{5} \mathrm{D}$ level, or the OI $927 \mathrm{~nm}$ line. As pointed out by Kiselman (1993), photon losses in the radiative transitions originating in these levels act to shift the equilibrium away from LTE to one where the upper levels are underpopulated and where the lower levels are overpopulated, analogous to the way the OI $777 \mathrm{~nm}$ lines act on the $3 \mathrm{p}{ }^{5} \mathrm{P}$ level and the $3 \mathrm{~s}{ }^{5} \mathrm{~S}$ level. There is thus a flow into the $3 \mathrm{p}{ }^{5} \mathrm{P}$ level which competes with the underpopulating effect of the OI $777 \mathrm{~nm}$ lines. This competing effect complicates the behaviour of the $3 \mathrm{p}{ }^{5} \mathrm{P}$ level and is what drives the overpopulation seen at $\log \tau_{500} \approx-1.0$ in the solar metallicity turn-off star in Fig. 2. In contrast, the behaviour of the $3 \mathrm{~s}{ }^{5} \mathrm{~S}$ level is simpler to understand. The OI $777 \mathrm{~nm}$ lines induce a flow into the level, which is metastable and can develop a relatively large overpopulation.

The relative size of these various effects are sensitive to the stellar parameters. Larger effective temperatures, smaller effective gravities and larger oxygen abundances tend to make the OI $777 \mathrm{~nm}$ lines stronger. which tends to shift the system further away from LTE. They also make the higher excitation lines (such as the OI $927 \mathrm{~nm}$ line) stronger. This increases the cascade effect described above, and is why the overpopulation in the $3 \mathrm{p}{ }^{5} \mathrm{P}$ levels is most prominent in the more metal rich turn-off stars.

The preceding discussion focused on the line forming regions of the OI $777 \mathrm{~nm}$ lines. At greater heights, the lines do not form efficiently because the populations of the high excitation levels are negligible in these low temperature regions. A discussion of the limiting behaviour of the departure coefficients and of $\rho_{i j}^{T}$ in these regions is therefore of lesser interest.

\subsection{D atmospheric inhomogeneities}

We now consider the effects of atmospheric inhomogeneities on the statistical equilibrium. We show in Fig. 4 the spatially-resolved gas temperature, the non-LTE frequencyintegrated contribution function for the absolute line flux depression, and departure coefficients of the $3 \mathrm{p}^{5} \mathrm{P}$ level and the $3 \mathrm{~s}{ }^{5} \mathrm{~S}$ level (i.e. the upper and lower levels of the OI $777 \mathrm{~nm}$ lines) on five maps of equal $\log \tau_{500}$ in a single $3 \mathrm{D}$ snapshot of a solar metallicity turn-off star. In Fig. 5 we show the same quantities, in a snapshot of a metal poor turn-off star.

At the largest depths shown ( $\log \tau_{500}=0.0$, corresponding roughly to the visible surface) the granulation pattern which results from convective turnover at the bottom of the photosphere (e.g. Magic et al. 2013a) is evident in the gas temperature maps. The bright granules occupying the largest area fraction are composed of hot upflowing gas; cooler downflowing gas make up the intergranular lanes. At the smallest depths shown $\left(\log \tau_{500}=-2.0\right)$, reverse granulation features (e.g. Cheung et al. 2007) are apparent in the gas temperature maps. The upflowing gas cools efficiently by adiabatic expansion, while radiative reheating and mechanical compression cause the downflowing gas to increase slightly in temperature (Magic et al. 2013b).

Most of the emergent OI $777 \mathrm{~nm}$ line formation (as indicated by the contribution function for the absolute line flux depression) occurs at depths $\log \tau_{500} \lesssim 0.5$, because photons from larger depths are unlikely to escape through the atmosphere (cf. the escape probability $\mathrm{e}^{-\tau}$ ). Around the optical surface $\left(-1.0 \lesssim \log \tau_{500} \lesssim 0.0\right)$, the line formation is associated with the granulation pattern, while at greater heights $\left(\log \tau_{500} \lesssim-1.5\right)$ in the solar metallicity example, line formation is associated with the reverse granulation features. (in the metal poor example (Fig. 5), line formation is inefficient on account of the low oxygen abundance.) These associations are because the line opacity of the high-excitation OI $777 \mathrm{~nm}$ lines is larger in regions of higher gas temperatures, due to greater excitation out of the oxygen ground level.

At large depths $\left(\log \tau_{500} \gtrsim 0.5\right)$, the departure coefficients stay close to unity because the large densities guarantee that collisional rates dominate over all radiative rates. Around the optical surface $\left(-1.0 \lesssim \log \tau_{500} \lesssim 0.0\right)$, the $3 \mathrm{~s}$ ${ }^{5} \mathrm{~S}$ level develops an overpopulation with respect to LTE in both figures; in Fig. 4 the $3 \mathrm{p}{ }^{5} \mathrm{P}$ level also becomes overpopulated. At greater heights $\left(\log \tau_{500} \lesssim-1.5\right)$ in Fig. 4 the overpopulations in the levels are associated with the reverse granulation features. This behaviour can be understood from the preceding discussion of the non-LTE mechanism (Sect. 3.1). The departures from LTE in the $3 \mathrm{~s}{ }^{5} \mathrm{~S}$ and $3 \mathrm{p}{ }^{5} \mathrm{P}$ levels are driven largely by photon losses in the high excitation OI $777 \mathrm{~nm}$ lines, and are also affected by photon losses in even higher excitation lines such as the OI $927 \mathrm{~nm}$ lines. The general trend of increasing departure coefficient with height is due to photon losses becoming more severe as the material becomes increasingly optically thin. On top of that, photon losses are more severe in regions of high temperature, where the lines are stronger; consequently there is a close association of the departure coefficients with the reverse granulation features.

The efficient line formation of the OI $777 \mathrm{~nm}$ lines in the reverse granulation is interesting because it drives an 


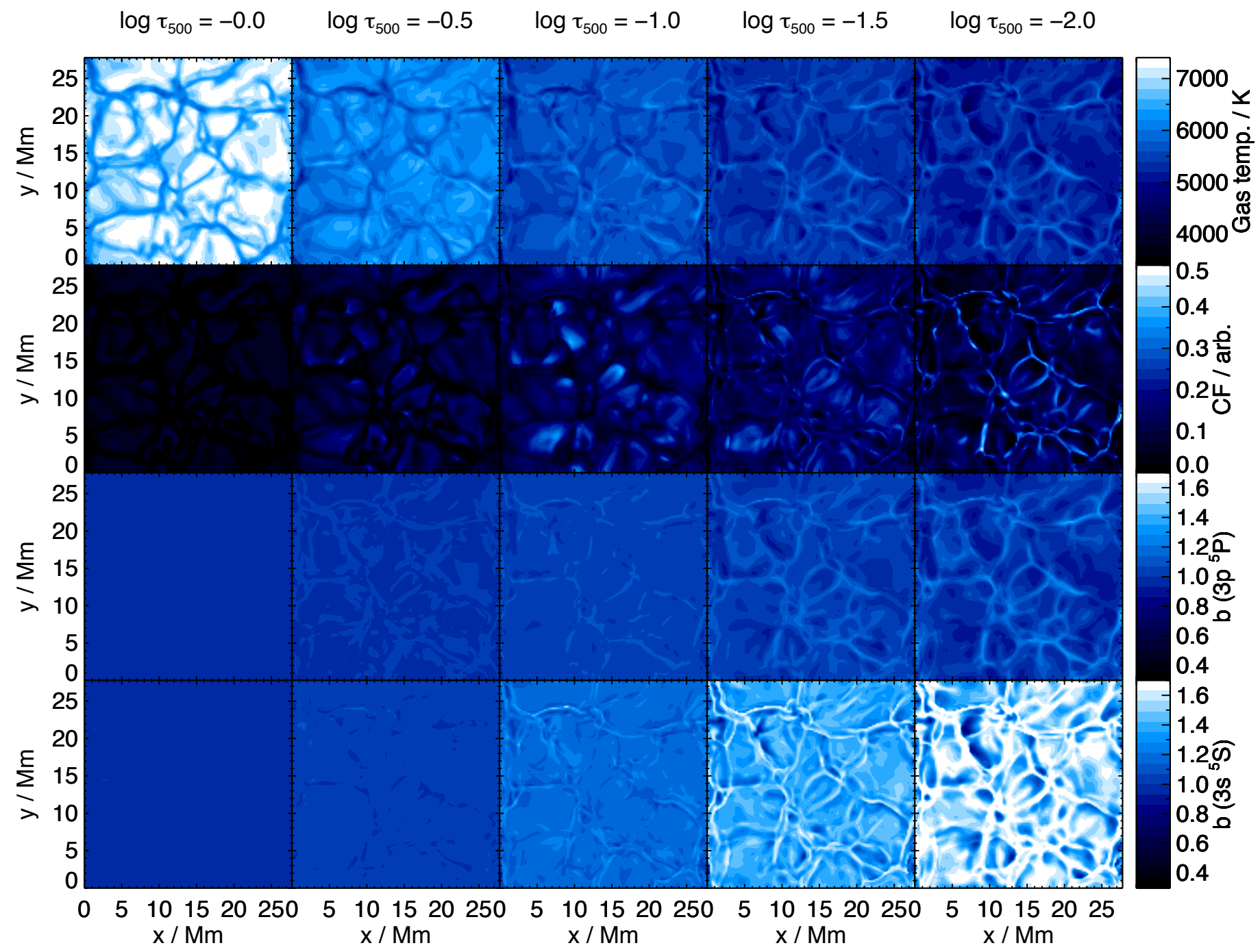

Figure 4. Surfaces of equal vertical optical depth $\log \tau_{500}$ in a snapshot of a turn-off star $\left(T_{\mathrm{eff}} \approx 6500 \mathrm{~K}, \log \left(\mathrm{g} / \mathrm{cm} \mathrm{s}^{-2}\right)=4.0\right)$ with $[\mathrm{Fe} / \mathrm{H}]=0.0$, and oxygen abundance $\log \epsilon_{\mathrm{O}}=8.7$. The quantities from top to bottom are gas temperature, non-LTE frequency-integrated contribution function for the absolute line flux depression (in arbitrary units), departure coefficient of the $3 \mathrm{p}^{5} \mathrm{P}$ level (the upper level of the OI $777 \mathrm{~nm}$ lines), and departure coefficient of the $3 \mathrm{~s}{ }^{5} \mathrm{~S}$ level (the lower level of the OI $777 \mathrm{~nm}$ lines). The mean formation depth $( \pm$ one standard deviation), as defined in Fig. 3 , is $\mathbf{E}\left[\log \tau_{500}\right] \approx-1.66( \pm 0.86)$.

overpopulation with respect to LTE in the $3 \mathrm{~s}{ }^{5} \mathrm{~S}$ level, which in turn promotes line formation. This is an example of the coupling of $3 \mathrm{D}$ effects with non-LTE effects.

\subsection{Non-vertical radiative transfer}

Non-local radiation along oblique rays from hotter or cooler environments can affect the statistical equilibrium in the line forming regions (e.g. Stenholm \& Stenflo 1977; Holzreuter $\&$ Solanki 2013). The influence of this is gauged by repeating the non-LTE calculations on the 3D model atmospheres, but treating the model as an ensemble of 1D columns, each column effectively extended in the horizontal directions to infinity (so called 1.5D non-LTE; e.g. Kiselman \& Nordlund 1995).

In Fig. 6 we show the spatially-resolved relative differ- ences in the departure coefficients of the $3 p{ }^{5} \mathrm{P}$ level and of the $3 \mathrm{~s}{ }^{5} \mathrm{~S}$ level (i.e. the upper and lower levels of the OI $777 \mathrm{~nm}$ lines) between the 1.5D non-LTE case and the 3D non-LTE case, in the same snapshot of the solar metallicity turn-off star shown in Fig. 4 . Note that the relative difference in the departure coefficients are the same as that in the populations, because the LTE populations (the divisors in Eq. 8) are a property of the background atmosphere, which is left unchanged.

At large depths the populations are close to their LTE values in $1.5 \mathrm{D}$ and in $3 \mathrm{D}$. Higher up at $\log \tau_{500}=-1.5$ (within the peak line forming region), the differences in the populations of the $3 \mathrm{~s}{ }^{5} \mathrm{~S}$ level are of the order $10 \%$, with the populations being larger in the $3 \mathrm{D}$ case. The differences are associated with the reverse granulation. In $1.5 \mathrm{D}$, the hot reverse granulation features (thin, bright filaments in the 


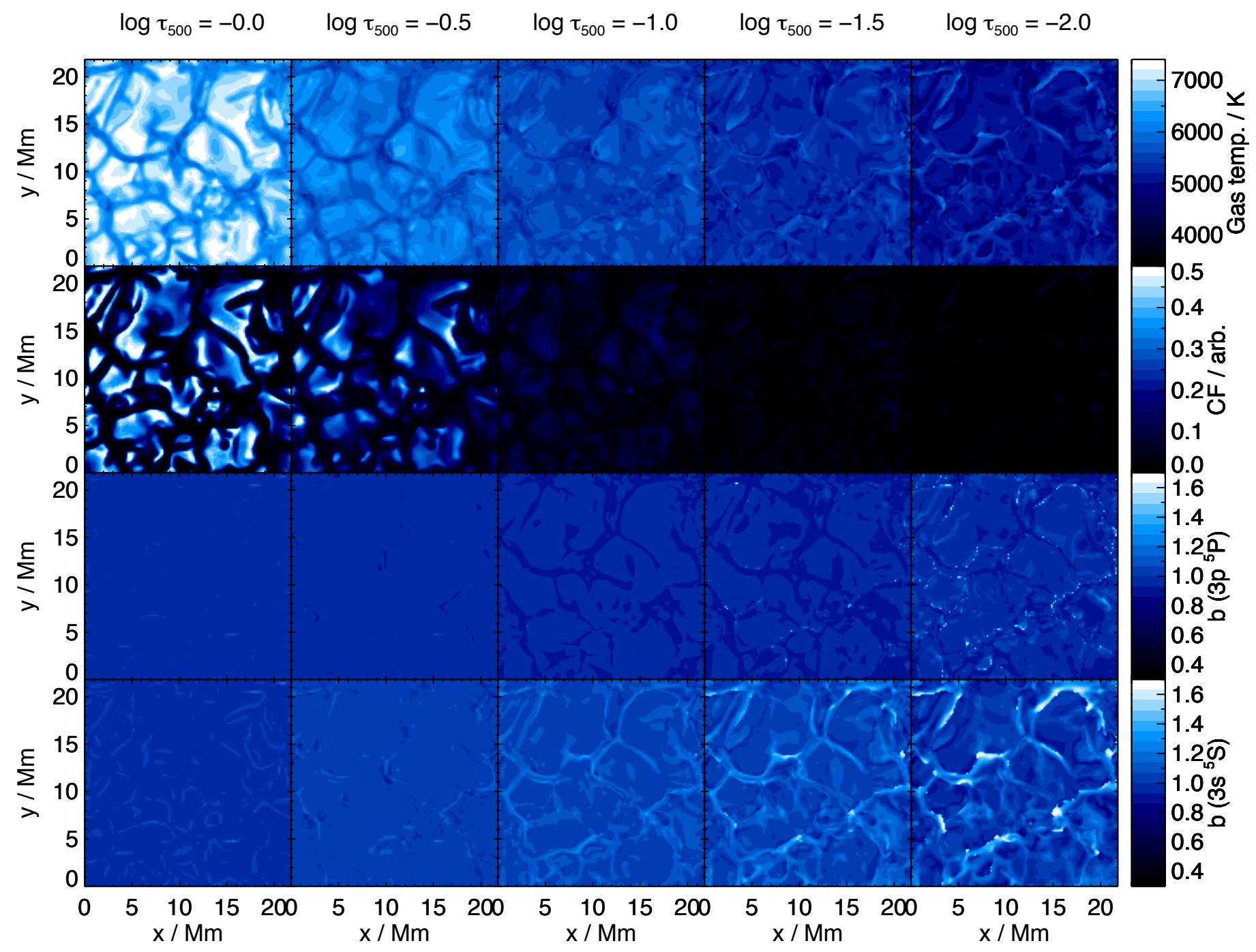

Figure 5. Surfaces of equal vertical optical depth $\log \tau_{500}$ in a snapshot of a turn-off $\operatorname{star}\left(T_{\text {eff }} \approx 6500 \mathrm{~K}, \log \left(\mathrm{g} / \mathrm{cm} \mathrm{s}^{-2}\right)=4.0\right)$ with $[\mathrm{Fe} / \mathrm{H}]=-3.0$, and oxygen abundance $\log \epsilon_{\mathrm{O}}=6.2$. The quantities from top to bottom are gas temperature, non-LTE frequencyintegrated contribution function for the absolute line flux depression (in arbitrary units), departure coefficient of the $3 \mathrm{p}{ }^{5} \mathrm{P}$ level (the upper level of the OI $777 \mathrm{~nm}$ lines), and departure coefficient of the $3 \mathrm{~s}{ }^{5} \mathrm{~S}$ level (the lower level of the OI $777 \mathrm{~nm}$ lines). The mean formation depth ( \pm one standard deviation), as defined in Fig. 3, is $\mathbf{E}\left[\log \tau_{500}\right] \approx-0.55( \pm 0.59)$.

plots) are infinitely extended in the horizontal directions, whereas in 3D these features are surrounded by cooler gas. More photons can be created when the temperature is larger (cf. the Planck function). Consequently, there are fewer nonlocal photons propagating into these hot features in 3D than in $1.5 \mathrm{D}$. With fewer photoexcitations, the statistical equilibrium in these regions is such that there is a larger overpopulation of $3 \mathrm{~s}{ }^{5} \mathrm{~S}$ level. In contrast, the mean radiation field in the surrounding regions (dark areas around the thin filaments) is larger in $3 \mathrm{D}$ than in $1.5 \mathrm{D}$. This is because extra non-local photons from the hot regions propagate into the cooler regions. With more photoexcitations in these cool regions, the $3 \mathrm{~s}{ }^{5} \mathrm{~S}$ level has a smaller population in $3 \mathrm{D}$ than in 1.5D. The behaviour of the $3 \mathrm{p}^{5} \mathrm{P}$ level is more complicated, since there is a flow from the highly excited levels into it via the OI $927 \mathrm{~nm}$ lines, that competes with the flow out of the level via the OI $777 \mathrm{~nm}$ lines.

The statistical equilibrium populations are slightly increased or decreased in $3 \mathrm{D}$ compared to $1.5 \mathrm{D}$ at different locations in the simulation box. This means that net effect of non-vertical radiative transfer on the averaged emergent fluxes is small. Effects are most severe in solar metallicity turn-off stars, in which the OI $777 \mathrm{~nm}$ lines form high up in the temperature inhomogeneities associated with reverse granulation play a significant role; Fig. 6 is indicative of the worst case.

We compare in Fig. 7 theoretical disk-integrated flux spectra in the vicinity of the OI $777 \mathrm{~nm}$ lines for a variety of test cases, including 1.5D non-LTE. (The final emergent intensities are computed using full $3 \mathrm{D}$ radiative transfer.) The 1.5D non-LTE line strength is of the order $0.01 \mathrm{dex}$ 


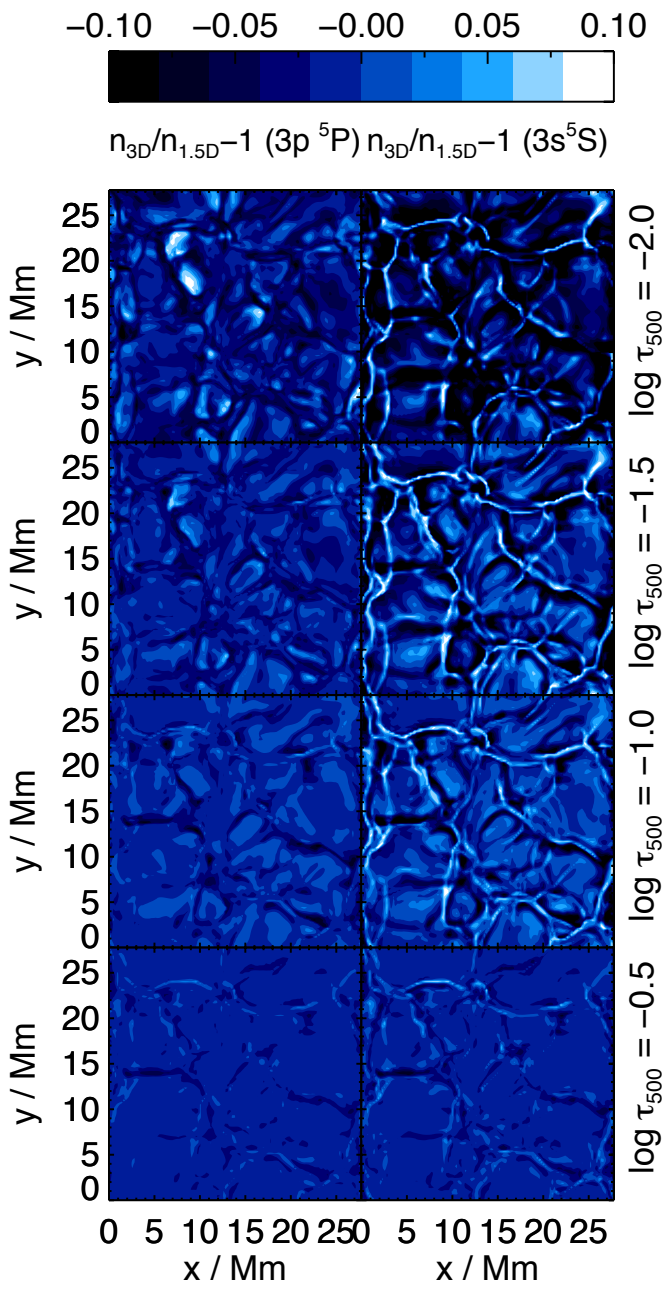

Figure 6. Relative difference in the departure coefficients of the $3 \mathrm{p}{ }^{5} \mathrm{P}$ level (left) and of the $3 \mathrm{~s}{ }^{5} \mathrm{~S}$ level (right) (i.e. the upper and lower levels of the OI $777 \mathrm{~nm}$ lines, respectively) between the $1.5 \mathrm{D}$ non-LTE case (which neglects non-vertical radiative transfer) and the 3D non-LTE case, on surfaces of equal vertical optical depth $\log \tau_{500}$ in a snapshot of a turn-off star $\left(T_{\text {eff }} \approx 6500 \mathrm{~K}\right.$, $\left.\log \left(\mathrm{g} / \mathrm{cm} \mathrm{s}^{-2}\right)=4.0\right)$ with $[\mathrm{Fe} / \mathrm{H}]=0.0$, and oxygen abundance $\log \epsilon_{\mathrm{O}}=8.7$.

larger than the standard 3D non-LTE case in the most extreme case (solar metallicity turn-off stars). This suggests the OI $777 \mathrm{~nm}$ lines could be modelled accurately and at considerably smaller computational cost by using efficient $1.5 \mathrm{D}$ non-LTE radiative transfer codes (e.g. Pereira \& Uitenbroek $2015)$ to compute the statistical equilibrium.

\subsection{Background UV opacity at low $[\mathrm{Fe} / \mathrm{H}]$}

Fabbian et al. (2009) reported large departures from LTE at low metallicities, associated with photon pumping in the UV resonance OI $130 \mathrm{~nm}$ lines. However, their analysis did not include the $121.5 \mathrm{~nm}$ Lyman- $\alpha$ transition as a background opacity, which is significant because the line is very strong and the OI $130 \mathrm{~nm}$ lines sit on its red wing. Neglecting Lyman- $\alpha$, we were able to reproduce the large non-LTE effects in the OI $777 \mathrm{~nm}$ lines; when this transition is included in the analysis however, the statistical equilibrium at low metallicities is much closer to LTE.

We compare in Fig. 8 the normalized net flow into the $3 \mathrm{~s}{ }^{3} \mathrm{~S}$ level (i.e. the upper level of the UV resonance OI $130 \mathrm{~nm}$ lines) when Lyman- $\alpha$ is included and when it is not. When Lyman- $\alpha$ is neglected, the background opacity in the UV is significantly lowered. This allows for an abundance of UV photons being absorbed via the UV resonance OI $130 \mathrm{~nm}$ lines, driving a large overpopulation with respect to LTE in the $3 \mathrm{~s}{ }^{3} \mathrm{~S}$ level. (The lower levels of these lines is the ground state of oxygen, which retain their LTE populations.) As can be seen in Fig. 8, and as discussed by Fabbian et al. (2009), collisional coupling between the $3 \mathrm{~s}^{3} \mathrm{~S}$ level and the $3 \mathrm{~s}{ }^{5} \mathrm{~S}$ level (i.e. the lower level of the OI $777 \mathrm{~nm}$ lines) propagates a flow from the former level over to the latter level. The overpopulation is then propagated to the $3 p$ ${ }^{5} \mathrm{P}$ level (i.e. the upper level of the OI $777 \mathrm{~nm}$ lines) by collisional coupling. This increases the OI $777 \mathrm{~nm}$ line opacity, leaving the source function only slightly perturbed.

The Lyman- $\alpha$ line provides an efficient alternative destruction route for UV photons that largely stifles the photon pumping effect in the OI $130 \mathrm{~nm}$ lines. Without the photon pumping in the OI $130 \mathrm{~nm}$ lines, the OI $777 \mathrm{~nm}$ line opacity is closer to its LTE value; in other words we find the statistical equilibrium to be much closer to LTE in the metal poor regime.

We compare in Fig. 7 theoretical flux spectra in the vicinity of the OI $777 \mathrm{~nm}$ lines when Lyman- $\alpha$ is included and when it is not. The importance of this effect is greatest in metal poor turn-off stars (second panel of Fig. 7), in which neglecting Lyman- $\alpha$ strengthens the OI $777 \mathrm{~nm}$ lines by of the order 0.75 dex (with the exact value depending on the choice of oxygen abundance). At lower effective temperatures and higher surface gravities, and particularly at higher metallicities, neglecting Lyman- $\alpha$ incurs a smaller error.

Other lines and continua in the UV providing background opacity were included in the analysis, since they can also play a role in determining the statistical equilibrium of oxygen in metal poor stars. For example, the Lyman$\beta$ line at $102.6 \mathrm{~nm}$ was included in the analysis. It overlaps with a number of other UV oxygen lines; the mechanism by which the statistical equilibrium is affected is similar to that described above for Lyman- $\alpha$ and the OI $130 \mathrm{~nm}$ lines. We emphasise however that Lyman- $\alpha$ appears to be the most important UV background opacity to include, at least for the metal poor stars studied in this work. For more discussion on the effects of Lyman- $\beta$ and of other background UV opacities on the statistical equilibrium we refer the reader to Sect. 3.1.2 of Fabbian et al. (2009).

\subsection{Neutral hydrogen collisions}

It is important to consider how the neutral hydrogen collisions affect the results, because the adopted recipes are very uncertain (e.g. Barklem et al. 2011).

The collisional transition with the largest effect on the OI $777 \mathrm{~nm}$ lines is that between the $3 \mathrm{p}{ }^{5} \mathrm{P}$ level and the $3 \mathrm{~s}$ ${ }^{5} \mathrm{~S}$ level (i.e. the upper and lower levels of the lines). Collisional coupling is responsible for an upwards flow between these two levels that compensates the downwards flow driven by the corresponding radiative transition (Fig. 3). Obtaining accurate neutral hydrogen collisional rate coefficients for 


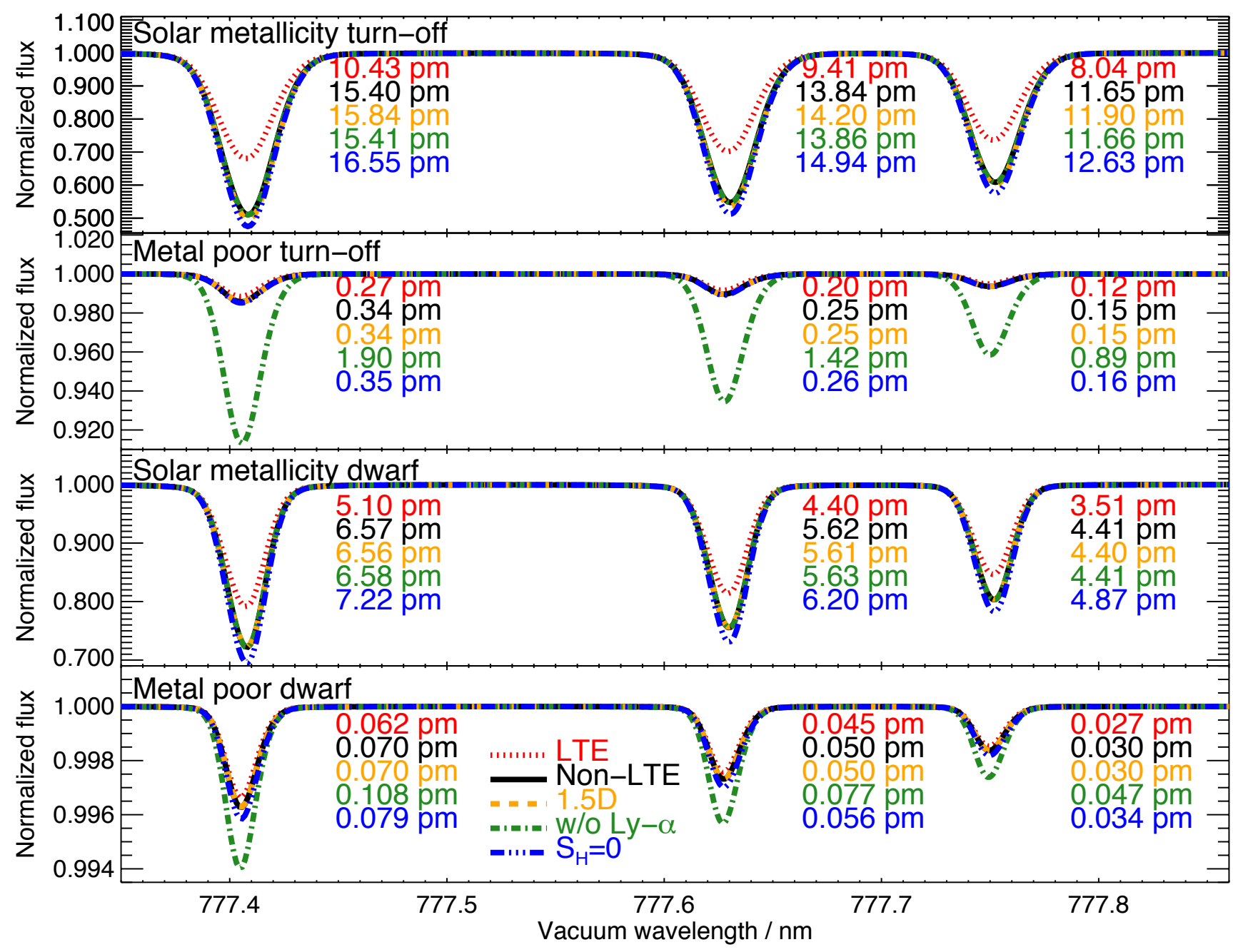

Figure 7. Normalized theoretical flux spectra (from Multi3D and ScATE, as described in Sect. 2.6) in the vicinity of the OI $777 \mathrm{~nm}$ lines in a turn-off star $\left(T_{\text {eff }} \approx 6500 \mathrm{~K}, \log \left(\mathrm{g} / \mathrm{cm} \mathrm{s}^{-2}\right)=4.0\right)$ and in a dwarf $\operatorname{star}\left(T_{\text {eff }} \approx 5500 \mathrm{~K}, \log \left(\mathrm{g} / \mathrm{cm} \mathrm{s}^{-2}\right)=4.0\right)$ with $[\mathrm{Fe} / \mathrm{H}]=0.0$, $\log \epsilon_{\mathrm{O}}=8.7$ (solar metallicity) and $[\mathrm{Fe} / \mathrm{H}]=-3.0, \log \epsilon_{\mathrm{O}}=6.2$ (metal poor). Spectra are shown for the 3D LTE, 3D non-LTE, 1.5D non-LTE (Sect. 3.3), 3D non-LTE without Lyman- $\alpha$ (Sect. 3.4), and 3D non-LTE with $S_{\mathrm{H}}=0.0$ (Sect. 3.5); their equivalent widths in $\mathrm{pm}=10^{-12} \mathrm{~m}$, obtained by direct integration, are indicated in that order to the right of each line. 1.5D refers to the radiative transfer scheme used to compute the statistical equilibrium; the final emergent intensities are (in all cases shown) computed using full 3D radiative transfer.

this transition are of vital importance. Of secondary importance are the rate coefficients for the collisional transitions between the highly excited upper levels (4s ${ }^{5} \mathrm{~S}$ and above). The close coupling between these levels is maintained by neutral hydrogen collisions, rather than electron collisions; errors in these rates will influence the downwards cascade into the $3 \mathrm{p}{ }^{5} \mathrm{P}$ level via the OI $927 \mathrm{~nm}$ line (see Sect. 3.1).

Fortunately, the LTE abundance errors for the OI $777 \mathrm{~nm}$ flux do not seem to be too sensitive to the hydrogen collisions. We show in Fig. 7 theoretical flux spectra in the vicinity of the OI $777 \mathrm{~nm}$ lines when $S_{\mathrm{H}}$ is set to zero, so that the collisions with neutral hydrogen are neglected entirely. In the cases shown, and more generally across the grid, neglecting hydrogen collisions strengthens the OI $777 \mathrm{~nm}$ lines by of the order 0.05 dex. It bears repeating that the main results of this work are calculated with $S_{\mathrm{H}}$ set to unity (i.e. no scaling of the rate coefficients), this choice being based on a solar analysis of the OI $777 \mathrm{~nm}$ lines (Sect. 2.4).

\subsection{Abundance errors}

Abundance errors (Sect. 2.6) are good indicators of the importance (or the lack of importance) of modelling the system using 3D stellar models and/or multi-level non-LTE radiative transfer. We show the abundance errors as functions of the different parameters $\left(T_{\text {eff }}, \log g,[\mathrm{Fe} / \mathrm{H}], \xi\right.$ and $\left.\log \epsilon_{\mathrm{O}}\right)$ in Fig. 9. The plots in each column illustrate how the abun- 


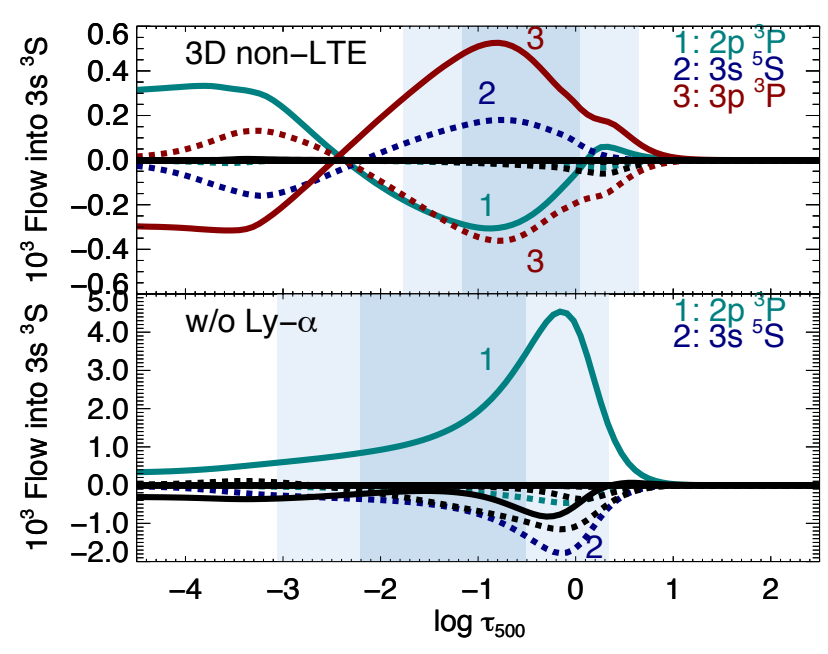

Figure 8. Normalized net flow into the $3 \mathrm{~s}{ }^{3} \mathrm{~S}$ level (the upper level of the OI $130 \mathrm{~nm}$ lines) at different depths in a metal poor turn-off $\operatorname{star}\left(T_{\text {eff }} \approx 5500 \mathrm{~K}, \log \left(\mathrm{g} / \mathrm{cm} \mathrm{s}^{-2}\right)=4.0,[\mathrm{Fe} / \mathrm{H}]=-3.0\right.$, $\left.\log \epsilon_{\mathrm{O}}=6.2\right)$ with and without Lyman- $\alpha$ background line opacity in the non-LTE analysis. Collisional transitions are indicated as dashed lines while radiative transitions are indicated as solid lines. Plotted here are median values on surfaces of equal $\log \tau_{500}$. The shaded areas correspond to the mean formation depth \pm one standard deviation, and \pm two standard deviations (with the mean formation depth and its standard deviation defined in Fig. 3).

dance errors depend on a single parameter, while the remaining four are fixed. The rows show 1D non-LTE abundance errors $\left(\Delta_{3 \mathrm{~N}}^{1 \mathrm{~N}}\right)$, 1D LTE abundance errors $\left(\Delta_{3 \mathrm{~N}}^{1 \mathrm{~L}}\right)$, and 3D LTE abundance errors $\left(\Delta_{3 \mathrm{~N}}^{3 \mathrm{~L}}\right)$.

The abundance errors were obtained by linear interpolation in the 1D non-LTE, 1D LTE, and 3D LTE abundances (respectively). The abundance errors also had to be interpolated linearly in the effective temperature, because the effective temperature grid is irregular (see Sect. 2.6).

Importantly, the abundance errors plotted in Fig. 9 are all relative to, and functions of, the $3 \mathrm{D}$ non-LTE oxygen abundance, enabling a direct comparison between them. Furthermore, the 1D plane-parallel hydrostatic Aтmo (Magic et al. 2013a, Appendix A) model atmospheres have as far as possible identical input physics to the STAGGER models (Sect. 2.5). This enables a fair comparison of the non-LTE effects in 1D and 3D.

We show the abundance errors for the [OI] $777 \mathrm{~nm}$ lines, the $[\mathrm{OI}] 636 \mathrm{~nm}$ and $[\mathrm{OI}] 630 \mathrm{~nm}$ lines, and the OI $616 \mathrm{~nm}$ line; these are discussed in turn.

\subsubsection{OI 7r7nm lines}

The OI $777 \mathrm{~nm}$ lines are very prone to non-LTE effects. As discussed in Sect. 3.1, photon losses in the high excitation OI $777 \mathrm{~nm}$ lines drive an overpopulation of the $3 \mathrm{~s}{ }^{5} \mathrm{~S}$ level (i.e. the lower level of these lines) with respect to LTE. The cascade effect, in which there is a flow from the highly excited upper oxygen levels into the the $3 p{ }^{5} \mathrm{P}$ level (i.e. the upper level of the OI $777 \mathrm{~nm}$ lines) via the OI $927 \mathrm{~nm}$ line, can also contribute to the overpopulation of the $3 \mathrm{~s}{ }^{5} \mathrm{~S}$ level.
Larger abundance errors (or more negative abundance corrections) can therefore be expected where the high excitation lines are stronger: i.e. where the effective temperature is larger, the surface gravity is smaller, and the oxygen abundance is larger (Fig. 9: third row; first, second and fifth columns). The blue component of these three lines has the largest intrinsic strength, so it typically has the largest non-LTE abundance differences. The red component is the weakest, so it typically has the smallest non-LTE abundance differences.

The abundance errors have a mild dependence on stellar metallicity (Fig. 9: third column). The trend reflects mild changes in the mean temperature stratification of the $3 \mathrm{D}$ models at fixed effective temperature: as $[\mathrm{Fe} / \mathrm{H}]$ is decreased, the gas temperature becomes cooler in the line forming regions, which results in smaller non-LTE effects.

The 1D non-LTE and 1D LTE abundance errors vary with 1D microturbulence parameter $\xi$ (Fig. 9: first and second rows; fourth column). When the OI $777 \mathrm{~nm}$ lines are strong, increasing this parameter increases the equivalent widths of the lines modelled in $1 \mathrm{D}$, while the equivalent widths of the lines modelled in $3 \mathrm{D}$ are fixed (the $3 \mathrm{D}$ grids being computed without any analogous microturbulence parameter). This reduces the $1 \mathrm{D}$ non-LTE and $1 \mathrm{D}$ LTE abundance errors. When the OI $777 \mathrm{~nm}$ lines are weak, they lose their sensitivity to the microturbulence parameter.

Although the 1D non-LTE abundance errors are sensitive to the choice of $1 \mathrm{D}$ microturbulence parameter, they are typically of the order 0.05 to 0.1 dex (Fig. 9: first row). In other words, the 3D models exacerbate the non-LTE effects; part of the reason is the efficient OI $777 \mathrm{~nm}$ line formation in the atmospheric inhomogeneities driving larger departures from LTE, as discussed in Sect. 3.2. This result has practical implications. Typically, surveys of oxygen abundances in FGK-type stars that are based on the OI $777 \mathrm{~nm}$ lines adopt the procedure of correcting 1D LTE abundances using precomputed 1D non-LTE abundance corrections (e.g. Ramírez et al. 2013; Nissen et al. 2014). Our 1D non-LTE abundance errors suggest that this procedure systematically overestimates the oxygen abundance, albeit by a rather small amount.

\subsubsection{OI $616 \mathrm{~nm}$ line}

The high-excitation OI $616 \mathrm{~nm}$ line, originating between the $4 \mathrm{~d}{ }^{5} \mathrm{D}$ level and the $3 \mathrm{p}{ }^{5} \mathrm{P}$ level, suffers only mildly from non-LTE effects; the largest 3D LTE abundance errors in our grid are of the order 0.05 dex (Fig. 9: third row). Like the high-excitation OI $777 \mathrm{~nm}$ lines, the departures from LTE are larger when the effective temperature is larger, the surface gravity is smaller, and when the oxygen abundance is larger. The line is more susceptible to 3D effects. 1D abundance errors are typically of the order 0.1 to 0.2 dex (Fig. 9: first and second rows).

\subsection{3 [OI] $630 \mathrm{~nm}$ and [OI] $636 \mathrm{~nm}$ lines}

The [OI] $630 \mathrm{~nm}$ and [OI] $636 \mathrm{~nm}$ lines do not suffer from non-LTE effects. (Fig. 9: third row). The lower levels of these lines is the vastly populated ground level of oxygen, which retains its LTE population. The upper levels of these lines 


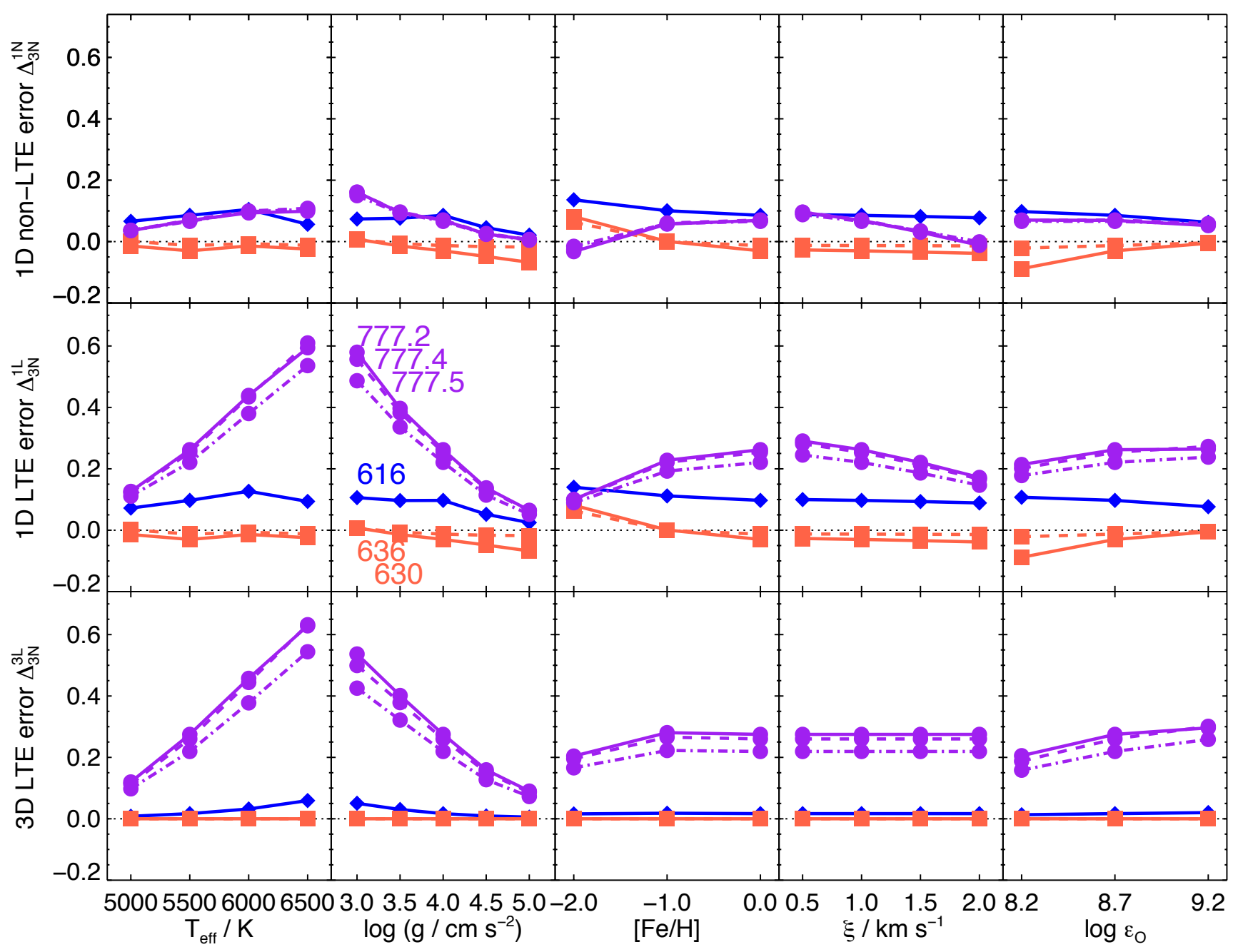

Figure 9. 1D non-LTE abundance errors $\left(\Delta_{3 \mathrm{~N}}^{1 \mathrm{~N}}\right)$, 1D LTE abundance errors $\left(\Delta_{3 \mathrm{~N}}^{1 \mathrm{~L}}\right)$, and 3D LTE abundance errors $\left(\Delta_{3 \mathrm{~N}}^{3 \mathrm{~L}}\right)$ for various atomic oxygen lines. The columns show how the abundance errors depend on a single parameter, with the other four parameters fixed. When the parameters are fixed, they have values $T_{\text {eff }}=5500 \mathrm{~K}, \log \left(\mathrm{g} / \mathrm{cm} \mathrm{s}^{-2}\right)=4.0,[\mathrm{Fe} / \mathrm{H}]=0.0,1 \mathrm{D}$ microturbulence $\xi=1.0 \mathrm{~km} \mathrm{~s}{ }^{-1}$, and $3 \mathrm{D}$ non-LTE oxygen abundance $\log \epsilon_{\mathrm{O}}=8.7$. Note that $\xi$ is only relevant in the first and second rows. The OI $616 \mathrm{~nm}$ line is denoted by diamonds connected with solid lines. The [OI] $630 \mathrm{~nm}$ and [OI] $636 \mathrm{~nm}$ lines are denoted by squares connected with solid and dashed lines, respectively. The [OI] $630 \mathrm{~nm}$ line includes the NiI blend; the adopted nickel abundance is solar scaled: $\log \epsilon_{\mathrm{Ni}}=6.20+[\mathrm{Fe} / \mathrm{H}]$ (Scott et al. 2015). Without the blend, the abundance errors for this line do not depart significantly from those for the [OI] $636 \mathrm{~nm}$ line. The three components of the OI $777 \mathrm{~nm}$ lines are labelled with their wavelengths in air and are denoted by circles connected with solid, dashed, and dot-dashed lines, from blue to red respectively.

are in close collisional coupling with the lower levels, ensuring that they too remain in LTE.

Like the OI $616 \mathrm{~nm}$ line, these lines are more susceptible to $3 \mathrm{D}$ effects. 1D abundance errors are most sensitive to the stellar metallicity, and tend to be larger in metal poor stars (Fig. 9: first and second rows; third column; the most severe case, $[\mathrm{Fe} / \mathrm{H}]=-3.0$, is not shown), in which the mean temperature stratification deviates more from the $1 \mathrm{D}$ counterparts (e.g. Nissen et al. 2002). In such stars, we find $1 \mathrm{D}$ abundance errors up to 0.15 dex.

For the $[\mathrm{OI}] 630 \mathrm{~nm}$ line we included the Ni blend (e.g. Lambert 1978; Allende Prieto et al. 2001; Johansson et al. 2003) in LTE and with a solar scaled nickel abundance: $\log \epsilon_{\mathrm{Ni}}=6.20+[\mathrm{Fe} / \mathrm{H}]$ (Scott et al. 2015). Any differences in the abundance errors between the [OI] $630 \mathrm{~nm}$ and [OI] $636 \mathrm{~nm}$ lines can be attributed to the different line strengths of the $\mathrm{Ni}$ blend in $1 \mathrm{D}$ and in $3 \mathrm{D}$, since the same nickel abundance was used in each case. Without the blend, the abundance differences for the [OI] $630 \mathrm{~nm}$ and $[\mathrm{OI}] 636 \mathrm{~nm}$ lines are indistinguishable from each other. The abundance corrections converge onto each other when oxygen, rather than nickel, dominates the $[\mathrm{OI}] 630 \mathrm{~nm}$ feature; for example, when the oxygen abundance is increased (Fig. 9: first and second rows; fifth column). 


\subsection{Grids of equivalent widths and abundance corrections}

We present in Table 4 predicted equivalent widths for the OI $616 \mathrm{~nm}$, [OI] $630 \mathrm{~nm}$, [OI] $636 \mathrm{~nm}$, and OI $777 \mathrm{~nm}$ lines, calculated on the nodes of the grid. Equivalent widths are shown for the four different solution schemes: 3D non-LTE, 3D LTE, 1D non-LTE, and 1D LTE. Abundance differences between any two schemes can be derived using the data in Table 4. For convenience, we provide in Table 5 the 3D nonLTE abundance corrections to 1D LTE based results $\left(\Delta_{1 \mathrm{~L}}^{3 \mathrm{~N}}\right)$, which are likely to be of most interest to the stellar spectroscopy community.

The data in Table 4 and Table 5 were interpolated and extrapolated so as to obtain equivalent widths and abundance corrections on regular grids of stellar parameters and input oxygen abundances. The regularly-spaced data are available upon request, and can also be found online ${ }^{5,6}$.

\section{COMPARISON WITH PREVIOUS 1D NON-LTE STUDIES}

3D non-LTE calculations for oxygen have previously been only performed for the Sun (Kiselman \& Nordlund 1995; Asplund et al. 2004; Pereira et al. 2009; Prakapavičius et al. 2013; Steffen et al. 2015). A detailed comparison with these papers plus a discussion of the solar oxygen abundance will be presented in a forthcoming paper. Here we restrict ourselves to comparing our 1D non-LTE results with those from several recently published studies: Takeda (2003); Ramírez et al. (2007); Fabbian et al. (2009); Sitnova et al. (2013). Since these studies provide 1D non-LTE abundance corrections $\left(\Delta_{1 \mathrm{~L}}^{1 \mathrm{~N}}\right)^{7}$, we compare abundance corrections, rather than abundance errors, in this section.

The ingredients of the analyses that are likely to have the most impact on the results are the model atom, in particular the description of electron collisions and hydrogen collisions (e.g. Przybilla et al. 2000; Barklem et al. 2011). Model atmospheres have a number of associated uncertainties, even in the 1D case (e.g. discussions in Castelli et al. 1997); therefore calculations over different families of model atmospheres can have systematically different outcomes. Missing background opacities, (e.g. Lyman- $\alpha$; see Sect. 3.4) can also be a source of error.

\subsection{Sitnova et al. (2013)}

The authors of this theoretical study performed calculations on AtLAs9 model atmospheres (Kurucz 1993a,b), and used Detail Butler \& Giddings (1985) to solve the statistical equilibrium. The authors updated the model atom of Przybilla et al. (2000), (which includes 51 OI levels plus the OII ground state, and all optically allowed bound-bound transitions) to use quantum mechanical electron excitation rate coefficients (Barklem 2007), and to include neutral hydrogen collisions following the recipe of Drawin $(1968,1969)$

5 http://www.mso.anu.edu.au/ ama51

6 http://inspect-stars.com

7 With the exception of Takeda (2003), in which $\Delta_{1 \mathrm{~N}}^{1 \mathrm{~L}}$ is provided. We interpolated their grid to obtain $\Delta_{1 \mathrm{~L}}^{1 \mathrm{~N}}$.

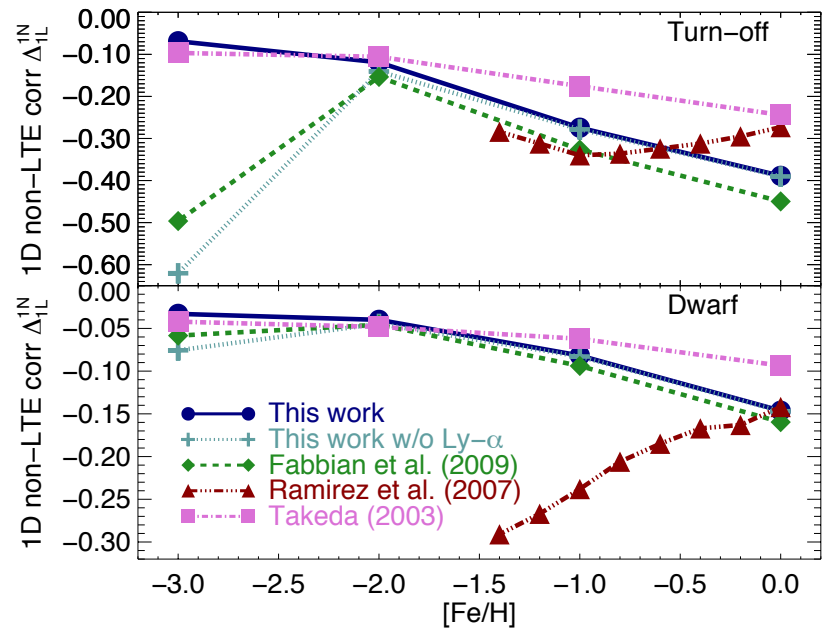

Figure 10. 1D non-LTE abundance corrections $\left(\Delta_{1 \mathrm{~L}}^{1 \mathrm{~N}}\right)$ for the middle OI $777 \mathrm{~nm}$ line, predicted by this work, Fabbian et al. (2009), Takeda (2003), and Ramírez et al. (2007). Also shown are the abundance corrections predicted by this work when background opacity from Lyman- $\alpha$ is not included. Top panel is for a turn-off star $\left(T_{\text {eff }}=6500 \mathrm{~K}, \log \left(\mathrm{g} / \mathrm{cm} \mathrm{s}^{-2}\right)=4.0\right)$, and the bottom panel is for a dwarf star $\left(T_{\text {eff }}=5500 \mathrm{~K}, \log \left(\mathrm{g} / \mathrm{cm} \mathrm{s}^{-2}\right)=\right.$ $4.0)$, with $1 \mathrm{D}$ microturbulence $\xi=1.0 \mathrm{~km} \mathrm{~s}^{-1}$ and varying chemical composition. The abundance corrections are functions of a measured LTE oxygen abundance which has a value of $8.7+[\mathrm{Fe} / \mathrm{H}]+[\alpha / \mathrm{Fe}]$, where the enhancement is $[\alpha / \mathrm{Fe}]=0.5$ for $[\mathrm{Fe} / \mathrm{H}] \leq-1.0,[\alpha / \mathrm{Fe}]=0.0$ for $[\mathrm{Fe} / \mathrm{H}]=0.0$, and a linear function in the region between them (i.e. $[\alpha / \mathrm{Fe}]=-0.5 \times[\mathrm{Fe} / \mathrm{H}]$ for $-1.0<[\mathrm{Fe} / \mathrm{H}]<0.0)$.

as formulated by Steenbock \& Holweger (1984) (and with $\left.S_{\mathrm{H}}=1.0\right)$; the treatment of collisional rates for radiativelyforbidden couplings is not discussed.

Included in their study is a small grid of $1 \mathrm{D}$ non-LTE abundance corrections $\left(\Delta_{1 \mathrm{~L}}^{1 \mathrm{~N}}\right)$ for the blue component of the OI $777 \mathrm{~nm}$ lines (Table 11 of Sitnova et al. 2013). Two of their calculations lie within our grid (Table 6); for these two cases we find good agreement with our corresponding abundance corrections.

\subsection{Fabbian et al. (2009)}

The authors performed calculations on MARCS model atmospheres (Gustafsson et al. 1975, and subsequent updates, prior to the most recent version of Gustafsson et al. (2008)), and Multi (Carlsson 1986), version 2.2, to solve the statistical equilibrium. This code has a number of features in common with the code we used (MuLTi3D); in particular the continuous opacity package is essentially the same. Their model atom (53 OI energy levels plus the ground state of OII, and 208 bound-bound transitions) is larger than that used here, but uses the same description for electron collisional rate coefficients (Barklem 2007), and neutral hydrogen collisional rate coefficients Lambert (1993). They perform calculations with $S_{\mathrm{H}}=0.0$ and with $S_{\mathrm{H}}=1.0$; only the latter case is discussed here. In particular, in this latter case the authors use the same value of $f_{\min }=10^{-3}$.

We show in Fig. 10 their 1D non-LTE abundance cor- 
Table 4. Predicted equivalent widths $W$ for different solution schemes "3N" (3D non-LTE), "3L" (3D LTE), "1N" (1D non-LTE), and "1L" (1D LTE), different oxygen lines labelled by one of "616", "630", "636", "7772", "7774", "7775", "777", and specified by the wavelength windows in air for direct integration $\lambda_{0}<\lambda<\lambda_{1}$, different sets of stellar parameters $\left(T_{\text {eff }}, \log g\right.$, [Fe/H], $\left.\xi\right)$, and different oxygen abundances $\log \epsilon_{\mathrm{O}}$. "7772", "7774" and "7775" refer to the three components of the OI $777 \mathrm{~nm}$ lines from blue to red respectively; " 777 " refers to all three components together. The [OI] $630 \mathrm{~nm}$ line contains the Ni blend in LTE and with a solar scaled nickel abundance: $\log \epsilon_{\mathrm{Ni}}=6.20+[\mathrm{Fe} / \mathrm{H}]$ (Scott et al. 2015). Only the first five rows are shown; the full table is available online.

\begin{tabular}{cccccccccc}
\hline Scheme & Line & $\lambda_{0} / \mathrm{nm}$ & $\lambda_{1} / \mathrm{nm}$ & $T_{\text {eff }} / \mathrm{K}$ & $\log \left(g / \mathrm{cm} \mathrm{s}^{-2}\right)$ & {$[\mathrm{Fe} / \mathrm{H}]$} & $\xi / \mathrm{km} \mathrm{s}^{-1}$ & $\log \epsilon_{\mathrm{O}}$ & $W / \mathrm{pm}$ \\
\hline \hline 3N & 616 & 615.73 & 615.97 & 4998 & 3.0 & -0.0 & 0.0 & 7.7 & 0.0330844 \\
\hline $3 \mathrm{~N}$ & 616 & 615.73 & 615.97 & 4998 & 3.0 & -0.0 & 0.0 & 8.2 & 0.0986540 \\
\hline $3 \mathrm{~N}$ & 616 & 615.73 & 615.97 & 4998 & 3.0 & -0.0 & 0.0 & 8.7 & 0.2713988 \\
\hline $3 \mathrm{~N}$ & 616 & 615.73 & 615.97 & 4998 & 3.0 & -0.0 & 0.0 & 9.2 & 0.6703377 \\
\hline $3 \mathrm{~N}$ & 616 & 615.73 & 615.97 & 4998 & 3.0 & -0.0 & 0.0 & 9.7 & 1.5235444 \\
\hline$\ldots$ & $\ldots$ & $\ldots$ & $\ldots$ & $\ldots$ & $\ldots$ & $\ldots$ & $\ldots$ & $\ldots$ & $\ldots$ \\
\hline
\end{tabular}

Table 5. 3D non-LTE abundance corrections to 1D LTE based results $\Delta_{1 \mathrm{~L}}^{3 \mathrm{~N}}$ (see Sect. 2.6) for different oxygen lines, labelled by one of "616", "630", "636", "7772", "7774", "7775", "777", and specified by the wavelength windows in air for direct integration $\lambda_{0}<\lambda<\lambda_{1}$, different sets of stellar parameters $\left(T_{\text {eff }}, \log g,[\mathrm{Fe} / \mathrm{H}], \xi\right)$, and different oxygen abundances $\log \epsilon_{\mathrm{O}}$. "7772", "7774" and "7775" refer to the three components of the OI $777 \mathrm{~nm}$ lines from blue to red respectively; " 777 " refers to all three components together. The [OI] $630 \mathrm{~nm}$ line contains the Ni blend in LTE and with a solar scaled nickel abundance: $\log \epsilon_{\mathrm{Ni}}=6.20+[\mathrm{Fe} / \mathrm{H}]$ (Scott et al. 2015). $\xi$ refers to the microturbulence parameter used in the 1D calculations; we emphasise that no microturbulence was used in any of the 3D calculations. Only the first five rows are shown; the full table is available online.

\begin{tabular}{ccccccccc}
\hline Line & $\lambda_{0} / \mathrm{nm}$ & $\lambda_{1} / \mathrm{nm}$ & $T_{\text {eff }} / \mathrm{K}$ & $\log \left(g / \mathrm{cm} \mathrm{s}^{-2}\right)$ & {$[\mathrm{Fe} / \mathrm{H}]$} & $\xi / \mathrm{km} \mathrm{s}^{-1}$ & $\log \epsilon_{\mathrm{O}}^{1 \mathrm{~L}}$ & $\Delta_{1 \mathrm{~L}}^{3 \mathrm{~N}}$ \\
\hline \hline 616 & 615.73 & 615.97 & 4998 & 3.0 & -0.0 & 0.5 & 8.2 & -0.1216693 \\
\hline 616 & 615.73 & 615.97 & 4998 & 3.0 & -0.0 & 0.5 & 8.7 & -0.1153454 \\
\hline 616 & 615.73 & 615.97 & 4998 & 3.0 & -0.0 & 0.5 & 9.2 & -0.1046884 \\
\hline 616 & 615.73 & 615.97 & 4998 & 3.0 & -0.0 & 0.5 & 9.7 & -0.0869795 \\
\hline 616 & 615.73 & 615.97 & 4996 & 3.0 & -1.0 & 0.5 & 7.2 & -0.1380291 \\
\hline$\ldots$ & $\ldots$ & $\ldots$ & $\ldots$ & $\ldots$ & $\ldots$ & $\ldots$ & $\ldots$ & $\ldots$ \\
\hline
\end{tabular}

Table 6. 1D non-LTE abundance corrections $\left(\Delta_{1 \mathrm{~L}}^{1 \mathrm{~N}}\right)$ for the blue component of the OI $777 \mathrm{~nm}$ lines in a solar metallicity turn-off star $\left(T_{\text {eff }}=6000 \mathrm{~K}, \log \left(\mathrm{g} / \mathrm{cm} \mathrm{s}^{-2}\right)=4.0\right)$ and a solar metallicity dwarf $\operatorname{star}\left(T_{\text {eff }}=5000 \mathrm{~K}, \log \left(\mathrm{g} / \mathrm{cm} \mathrm{s}^{-2}\right)=4.0\right)$ with $\xi=2.0 \mathrm{~km} \mathrm{~s}{ }^{-1}$ and 1D LTE oxygen abundance $\log \epsilon_{\mathrm{O}}=8.83$. We show results from Table 11 of Sitnova et al. (2013) for which the Barklem (2007) collisional rate coefficients are not scaled, results from the $S_{\mathrm{H}}=1.0$ grid of Fabbian et al. (2009), results from the grid described in Ramírez et al. (2007), and results interpolated from Table 2 of Takeda (2003). Note that the abundance corrections of Fabbian et al. (2009) and Ramírez et al. (2007) have no nominal dependence on $\xi$.

\begin{tabular}{cccccc}
\hline Star & This work & Sitnova et al. (2013) & Fabbian et al. (2009) & Ramírez et al. (2007) & Takeda (2003) \\
\hline \hline Turn-off & -0.31 & -0.34 & -0.35 & -0.26 & -0.19 \\
\hline Dwarf & -0.09 & -0.10 & -0.11 & -0.11 & -0.06 \\
\hline
\end{tabular}

rections $\left(\Delta_{1 \mathrm{~L}}^{1 \mathrm{~N}}\right)$ for the middle OI $777 \mathrm{~nm}$ line with $S_{\mathrm{H}}=1.0$, in turn-off stars and dwarf stars with varying chemical compositions. At $[\mathrm{Fe} / \mathrm{H}] \gtrsim-2.0$, their abundance corrections and our own are in good agreement. The turn-off star at $[\mathrm{Fe} / \mathrm{H}]=0.0$ represents the most discrepant case, where the 1D non-LTE abundance corrections differ by about 0.06 dex. The small discrepancies may be associated with dif- ferences between the MARCS model atmospheres and the ATMO model atmospheres..

At $[\mathrm{Fe} / \mathrm{H}] \lesssim-2.0$, the difference between our results and theirs is very large, and is due to the background UV opacity effect we discussed in Sect. 3.4. Their analysis did not include the Lyman- $\alpha$ transition as a background opacity. We re-ran our 1D non-LTE calculations with Lyman- $\alpha$ omitted. As seen in Fig. 10, the agreement between the two 
studies significantly improves, and the trend with $[\mathrm{Fe} / \mathrm{H}]$ is reproduced. This strongly suggests that the very negative abundance corrections found by Fabbian et al. (2009) at low $[\mathrm{Fe} / \mathrm{H}]$ would be quenched by including background UV opacity from the Lyman- $\alpha$ transition.

\subsection{Ramírez et al. (2007)}

The authors performed calculations on ATLAS9 model atmospheres (Kurucz 1993a,b), used TLusty(Hubeny 1988) to solve the statistical equilibrium and SyNSPEC(Hubeny et al. 1985) to generate emergent spectra. Their model atom was that of Allende Prieto et al. (2003) (54 OI levels plus the ground state of OII, and 242 bound-bound transitions). This study being antecedent to the quantum mechanical calculations of Barklem (2007), the authors used the classical prescriptions of van Regemorter (1962) and Seaton (1962), for radiatively allowed and forbidden electron excitation rate coefficients, respectively. The authors neglected neutral hydrogen collisions. Instead they apply a small empirical correction to their results, independent of stellar parameters, making their $1 \mathrm{D}$ non-LTE abundance corrections $\left(\Delta_{1 \mathrm{~L}}^{1 \mathrm{~N}}\right)$ less negative by 0.0355 dex and 0.018 dex for the blue component and the middle component of the OI $777 \mathrm{~nm}$ triplet lines.

Their grid of abundance corrections is finely spaced in metallicity but only extends down to $[\mathrm{Fe} / \mathrm{H}]=-1.4$. We compare their abundance corrections with our own in Table 6 and Fig. 10. The agreement is generally poor. The trend of their abundance corrections with $[\mathrm{Fe} / \mathrm{H}]$ is not qualitatively consistent with the trends from other studies shown on that plot. There is a strong sensitivity to the stellar metallicity that we do not find in our non-LTE results.

This discrepancy likely arises from more than one place. We note that missing background continuum opacities and background line opacities in the UV could drive photon pumping in the OI $130 \mathrm{~nm}$ lines and lead to large nonLTE effects in the system (cf. the mechanism described in Sect. 3.4); this would also explain the strong dependence on the stellar metallicity.

\subsection{Takeda (2003)}

A detailed description of their simulation setup can be found in Takeda $(1991,1992,2003)$. The author performed calculations on ATLAS9 models (Kurucz 1993a,b), using a large model atom (87 OI levels and 277 bound-bound transitions). The author used the classical prescriptions of van Regemorter (1962) and Auer \& Mihalas (1973) for the radiatively-allowed and radiatively-forbidden electron excitation rate coefficients. For the analogous neutral hydrogen rate coefficients they used the recipe of Drawin $(1968,1969)$ as formulated by Steenbock \& Holweger (1984) (and with $S_{\mathrm{H}}=1.0$ ), and modified the formula in Auer \& Mihalas (1973) for the radiatively-forbidden couplings.

Using their Table 2, we compare Takeda (2003) 1D nonLTE abundance corrections $\left(\Delta_{1 \mathrm{~L}}^{1 \mathrm{~N}}\right)$ with our own in Table 6 and Fig. 10. In contrast to what was found with Fabbian et al. (2009), we find very good agreement towards lower metallicities, and less good agreement at solar metallicity, with a discrepancy in the latter case that can be upwards of 0.15 dex. The good agreement at low metallicity reflects the fact that the non-LTE effects in the OI $777 \mathrm{~nm}$ lines are driven by the strength of the lines themselves, so as they get weaker the departures from LTE must get less severe. We note that Takeda (2003) have included the Lyman- $\alpha$ line in their analysis (see the discussion in $\S 4.3$ of Fabbian et al. 2009).

The discrepancies at higher metallicities are likely because of the different electron collisional rate coefficients used. As demonstrated by Fabbian et al. (2009) and Sitnova et al. (2013), the new rate coefficients by Barklem (2007) drive larger departures from LTE.

\section{CONCLUSIONS}

We have studied non-LTE atomic oxygen line formation across a grid of theoretical 3D hydrodynamic STAGGER model atmospheres of turn-off stars, dwarfs and subgiants. The main findings are as follows.

- Non-LTE effects on the OI $777 \mathrm{~nm}$ lines are mostly driven by photon losses in the lines themselves, which means their impact are largest when the lines themselves are stronger (i.e. at larger effective temperatures, smaller surface gravities, and larger oxygen abundances). 1D LTE abundances and 3D LTE abundances are over 0.2 dex too large, reaching 0.6 dex or more for solar metallicity turn-off stars.

- 3D effects (mean temperature stratification, atmospheric inhomogeneities) exacerbate the non-LTE effects. Consequently, 1D non-LTE abundances can be of the order 0.05 to 0.1 dex too large, with the exact error depending on the adopted 1D microturbulence.

- Non-vertical radiative transfer (another 3D effect) changes the OI $777 \mathrm{~nm}$ disk-integrated line strengths by only $0.01 \mathrm{dex}$. This suggests that these lines can be modelled accurately in $1.5 \mathrm{D}$ non-LTE.

- It is crucial to include all UV background opacities. Neglecting the Lyman- $\alpha$ transition introduces a spurious oxygen non-LTE mechanism (photon pumping in the OI $130 \mathrm{~nm}$ UV resonance lines) which increases the OI $777 \mathrm{~nm}$ line strengths by up to $0.75 \mathrm{dex}$ in metal poor turn-off stars.

- The treatment of neutral hydrogen collisions remains a deep uncertainty in the study of oxygen line formation. Neglecting hydrogen collisions increases the non-LTE OI $777 \mathrm{~nm}$ line strengths by of the order 0.05 dex.

- The OI $616 \mathrm{~nm}$ line suffers mildly from non-LTE effects, and the [OI] $630 \mathrm{~nm}$ and [OI] $636 \mathrm{~nm}$ lines do not show any significant departures from LTE. These lines however should be modelled in $3 \mathrm{D}$, with $1 \mathrm{D}$ abundance errors of the order 0.2 dex in the worst cases.

We have provided predicted 3D non-LTE based equivalent widths and abundance corrections to 1D LTE based results for the OI $616 \mathrm{~nm},[\mathrm{OI}] 630 \mathrm{~nm},[\mathrm{OI}] 636 \mathrm{~nm}$ lines, and OI $777 \mathrm{~nm}$ lines (Sect. 3.7: Table 4 and Table 5). These results should be useful to spectroscopic surveys of FGK-type stars. 


\section{ACKNOWLEDGEMENTS}

We thank Ivan Ramrez for providing his abundance differences and interpolation routines, which were used in Table 6 and Fig. 10, and Karin Lind for helping with uploading the results onto the online non-LTE database INSPECT. AMA and MA are supported by the Australian Research Council (ARC) grant FL110100012. RC acknowledges support from the ARC through DECRA grant DE120102940. This research was undertaken with the assistance of resources from the National Computational Infrastructure (NCI), which is supported by the Australian Government.

\section{REFERENCES}

Albrow M. D., Cottrell P. L., 1996, MNRAS, 278, 337

Allen C. W., 1973, Astrophysical quantities. London: University of London, Athlone Press, —c1973, 3rd ed., Ch. 3, Pg. 42

Allende Prieto C., Lambert D. L., Asplund M., 2001, ApJ, 556, L63

Allende Prieto C., Lambert D. L., Hubeny I., Lanz T., 2003, ApJS, 147, 363

Altrock R. C., 1968, Sol. Phys., 5, 260

Amarsi A. M., 2015, MNRAS, 452, 1612

Amarsi A. M., Asplund M., Collet R., Leenaarts J., 2015, MNRAS, 454, L11

Asplund M., 2005, ARA\&A, 43, 481

Asplund M., García Pérez A. E., 2001, A\&A, 372, 601

Asplund M., Grevesse N., Sauval A. J., Allende Prieto C., Kiselman D., 2004, A\&A, 417, 751

Asplund M., Grevesse N., Sauval A. J., Scott P., 2009, ARA\&A, 47,481

Asplund M., Nordlund Å., Trampedach R., Stein R. F., 1999, A\&A, 346, L17

Auer L., 1991, in Crivellari L., Hubeny I., Hummer D. G., eds, NATO Advanced Science Institutes (ASI) Series C Vol. 341 of NATO Advanced Science Institutes (ASI) Series C, Acceleration of Convergence. p. 9

Auer L., 2003, in Hubeny I., Mihalas D., Werner K., eds, Stellar Atmosphere Modeling Vol. 288 of Astronomical Society of the Pacific Conference Series, Formal Solution: EXPLICIT Answers. p. 3

Auer L. H., Mihalas D., 1973, ApJ, 184, 151

Barklem P. S., 2007, A\&A, 462, 781

Barklem P. S., Anstee S. D., O'Mara B. J., 1998, Publications of the Astron. Soc. of Australia, 15, 336

Barklem P. S., Belyaev A. K., Guitou M., Feautrier N., Gadéa F. X., Spielfiedel A., 2011, A\&A, 530, A94

Bergemann M., Lind K., Collet R., Magic Z., Asplund M., 2012, MNRAS, 427, 27

Boesgaard A. M., King J. R., Deliyannis C. P., Vogt S. S., 1999, AJ, 117, 492

Böhm-Vitense E., 1958, Zeitschrift fuer Astrophysik, 46, 108

Bond H. E., Nelan E. P., VandenBerg D. A., Schaefer G. H., Harmer D., 2013, ApJ, 765, L12

Bruls J. H. M. J., Vollmöller P., Schüssler M., 1999, A\&A, 348, 233

Butler K., Giddings J., 1985, University of London

Caffau E., Ludwig H.-G., Steffen M., Ayres T. R., Bonifacio P., Cayrel R., Freytag B., Plez B., 2008, A\&A, 488, 1031

Carlson B. G., 1963, Methods in Computational Physics, 1, 1

Carlsson M., 1986, Uppsala Astronomical Observatory Reports, 33

Carlsson M., Judge P. G., 1993, ApJ, 402, 344

Castelli F., Gratton R. G., Kurucz R. L., 1997, A\&A, 318, 841
Cheung M. C. M., Schüssler M., Moreno-Insertis F., 2007, A\&A, 461, 1163

Collet R., Asplund M., Thévenin F., 2005, A\&A, 442, 643

Collet R., Asplund M., Trampedach R., 2007, A\&A, 469, 687

Collet R., Magic Z., Asplund M., 2011, Journal of Physics Conference Series, 328, 012003

Cunto W., Mendoza C., Ochsenbein F., Zeippen C. J., 1993, A\&A, 275, L5

de Jager C., Neven L., 1967, Sol. Phys., 1, 27

Drawin H.-W., 1968, Zeitschrift fur Physik, 211, 404

Drawin H. W., 1969, Zeitschrift fur Physik, 225, 483

Eriksson K., Toft S. C., 1979, A\&A, 71, 178

Fabbian D., Asplund M., Barklem P. S., Carlsson M., Kiselman D., 2009, A\&A, 500, 1221

Fabiani Bendicho P., 2003, in Hubeny I., Mihalas D., Werner K., eds, Stellar Atmosphere Modeling Vol. 288 of Astronomical Society of the Pacific Conference Series, Basic Multidimensional Radiative Transfer. p. 419

Freytag B., Steffen M., Ludwig H.-G., Wedemeyer-Böhm S., Schaffenberger W., Steiner O., 2012, Journal of Computational Physics, 231, 919

Gratton R. G., Carretta E., Eriksson K., Gustafsson B., 1999, A\&A, 350, 955

Gustafsson B., Bell R. A., Eriksson K., Nordlund A., 1975, A\&A, 42, 407

Gustafsson B., Edvardsson B., Eriksson K., Jørgensen U. G., Nordlund Å., Plez B., 2008, A\&A, 486, 951

Hayek W., Asplund M., Carlsson M., Trampedach R., Collet R., Gudiksen B. V., Hansteen V. H., Leenaarts J., 2010, A\&A, 517, A49

Heiter U., Barklem P., Fossati L., Kildiyarova R., Kochukhov O., Kupka F., Obbrugger M., Piskunov N., Plez B., Ryabchikova T., Stempels H. C., Stütz C., Weiss W. W., 2008, Journal of Physics Conference Series, 130, 012011

Hildebrand F. B., 1956, Introduction to numerical analysis. International Series in Pure and Applied Mathematics, New York: McGraw-Hill, —c1956

Holzreuter R., Solanki S. K., 2013, A\&A, 558, A20

Hubeny I., 1988, Computer Physics Communications, 52, 103

Hubeny I., Mihalas D., 2014, Theory of Stellar Atmospheres. Princeton Univ. Press, Princeton, NJ

Hubeny I., Stefl S., Harmanec P., 1985, Bulletin of the Astronomical Institutes of Czechoslovakia, 36, 214

Ibgui L., Hubeny I., Lanz T., Stehlé C., 2013, A\&A, 549, A126

Israelian G., García López R. J., Rebolo R., 1998, ApJ, 507, 805

Jefferies J. T., 1966, in Hubenet H., ed., Abundance Determinations in Stellar Spectra Vol. 26 of IAU Symposium, The Solar Abundance of Iron. p. 207

Johansson S., Litzén U., Lundberg H., Zhang Z., 2003, ApJ, 584, L107

Kao C.-Y. J., Auer L. H., 1990, Monthly Weather Review, 118, 1551

Kiselman D., 1993, A\&A, 275, 269

Kiselman D., Nordlund A., 1995, A\&A, 302, 578

Kramida A., Yu. Ralchenko Reader J., and NIST ASD Team, 2015, NIST, NIST Atomic Spectra Database (ver. 5.3), [Online]. Available: http://physics.nist.gov/asd [2015, November 2]. National Institute of Standards and Technology, Gaithersburg, MD.

Krauss L. M., Chaboyer B., 2003, Science, 299, 65

Kurucz R., 1993a, ATLAS9 Stellar Atmosphere Programs and $2 \mathrm{~km} / \mathrm{s}$ grid. Kurucz CD-ROM No. 13. Cambridge, Mass.: Smithsonian Astrophysical Observatory, 1993., 13

Kurucz R. L., 1993b, in Dworetsky M. M., Castelli F., Faraggiana R., eds, IAU Colloq. 138: Peculiar versus Normal Phenomena in A-type and Related Stars Vol. 44 of Astronomical Society of the Pacific Conference Series, A New Opacity-Sampling Model Atmosphere Program for Arbitrary Abundances. p. 87 
Lambert D. L., 1978, MNRAS, 182, 249

Lambert D. L., 1993, Physica Scripta Volume T, 47, 186

Leenaarts J., Carlsson M., 2009, in Lites B., Cheung M., Magara T., Mariska J., Reeves K., eds, The Second Hinode Science Meeting: Beyond Discovery-Toward Understanding Vol. 415 of Astronomical Society of the Pacific Conference Series, MULTI3D: A Domain-Decomposed 3D Radiative Transfer Code. p. 87

Madhusudhan N., 2012, ApJ, 758, 36

Magain P., 1986, A\&A, 163, 135

Magic Z., Collet R., Asplund M., Trampedach R., Hayek W., Chiavassa A., Stein R. F., Nordlund Å., 2013a, A\&A, 557, A26

Magic Z., Collet R., Hayek W., Asplund M., 2013b, A\&A, 560, A8

McWilliam A., 1997, ARA\&A, 35, 503

Mihalas D., Athay R. G., 1973, ARA\&A, 11, 187

Nissen P. E., Chen Y. Q., Carigi L., Schuster W. J., Zhao G., 2014, A\&A, 568, A25

Nissen P. E., Primas F., Asplund M., Lambert D. L., 2002, A\&A, 390,235

Nordlund Å., Stein R. F., Asplund M., 2009, Living Reviews in Solar Physics, 6, 2

Olson G. L., Kunasz P. B., 1987, Journal of Quantitiative Spectroscopy and Radiative Transfer, 38, 325

Peach G., 1971, Journal of Physics B Atomic Molecular Physics, 4, 1670

Pecker J. C., 1959, Annales d'Astrophysique, 22, 499

Pereira T. M. D., Asplund M., Collet R., Thaler I., Trampedach R., Leenaarts J., 2013, A\&A, 554, A118

Pereira T. M. D., Asplund M., Kiselman D., 2009, A\&A, 508, 1403

Pereira T. M. D., Uitenbroek H., 2015, A\&A, 574, A3

Petigura E. A., Marcy G. W., 2011, ApJ, 735, 41

Piskunov N. E., Kupka F., Ryabchikova T. A., Weiss W. W., Jeffery C. S., 1995, A\&AS, 112, 525

Prakapavičius D., Steffen M., Kučinskas A., Ludwig H.-G., Freytag B., Caffau E., Cayrel R., 2013, Memorie della Societa Astronomica Italiana Supplementi, 24, 111

Przybilla N., Butler K., Becker S. R., Kudritzki R. P., Venn K. A., 2000, A\&A, 359, 1085

Ramírez I., Allende Prieto C., Lambert D. L., 2007, A\&A, 465, 271

Ramírez I., Allende Prieto C., Lambert D. L., 2013, ApJ, 764, 78

Rutten R. J., 2003, Radiative Transfer in Stellar Atmospheres, 8th edn.. Utrecht University

Rybicki G. B., Hummer D. G., 1991, A\&A, 245, 171

Rybicki G. B., Hummer D. G., 1992, A\&A, 262, 209

Saad Y., 2003, Iterative methods for sparse linear systems. Siam

Scharmer G. B., Bjelksjo K., Korhonen T. K., Lindberg B., Petterson B., 2003, in Keil S. L., Avakyan S. V., eds, Innovative Telescopes and Instrumentation for Solar Astrophysics Vol. 4853 of Society of Photo-Optical Instrumentation Engineers (SPIE) Conference Series, The 1-meter Swedish solar telescope. pp 341-350

Scott P., Asplund M., Grevesse N., Bergemann M., Sauval A. J., 2015, A\&A, 573, A26

Seaton M. J., 1962, in Bates D. R., ed., Atomic and Molecular Processes The Theory of Excitation and Ionization by Electron Impact. p. 375

Sedlmayr E., 1974, A\&A, 31, 23

Sitnova T. M., Mashonkina L. I., Ryabchikova T. A., 2013, Astronomy Letters, 39, 126

Stancil P. C., Schultz D. R., Kimura M., Gu J.-P., Hirsch G., Buenker R. J., 1999, A\&AS, 140, 225

Stasińska et al. G., ed. 2012, Oxygen in the Universe Vol. 54 of EAS Publications Series

Steenbock W., Holweger H., 1984, A\&A, 130, 319
Steffen M., Prakapavičius D., Caffau E., Ludwig H.-G., Bonifacio P., Cayrel R., Kučinskas A., Livingston W. C., 2015, arXiv: 1508.03487

Stenholm L. G., Stenflo J. O., 1977, A\&A, 58, 273

Storey P. J., Zeippen C. J., 2000, MNRAS, 312, 813

Takeda Y., 1991, A\&A, 242, 455

Takeda Y., 1992, PASJ, 44, 309

Takeda Y., 2003, A\&A, 402, 343

Thomas R. N., 1957, ApJ, 125, 260

Tinsley B. M., 1979, ApJ, 229, 1046

Uitenbroek H., 2001, ApJ, 557, 389

van Regemorter H., 1962, ApJ, 136, 906

VandenBerg D. A., Bergbusch P. A., Dotter A., Ferguson J. W., Michaud G., Richer J., Proffitt C. R., 2012, ApJ, 755, 15 